\title{
Parametric resonance of Josephson plasma waves: A theory for optically amplified interlayer superconductivity in $\mathrm{YBa}_{2} \mathrm{Cu}_{3} \mathrm{O}_{6+x}$
}

\author{
Marios H. Michael, ${ }^{1,{ }^{*}}$ Alexander von Hoegen, ${ }^{2}$ Michael Fechner, ${ }^{2}$ Michael Först, ${ }^{2}$ Andrea Cavalleri, ${ }^{2,3}$ and Eugene Demler ${ }^{1}$ \\ ${ }^{1}$ Department of Physics, Harvard University, Cambridge, Massachusetts 02138, USA \\ ${ }^{2}$ Max Planck Institute for the Structure and Dynamics of Matter, Hamburg, Germany \\ ${ }^{3}$ Department of Physics, University of Oxford, Oxford OX1 3PJ, United Kingdom
}

(Received 20 May 2020; revised 12 September 2020; accepted 14 September 2020; published 18 November 2020)

\begin{abstract}
Nonlinear interactions between collective modes play a definitive role in far out of equilibrium dynamics of strongly correlated electron systems. Understanding and utilizing these interactions is crucial to photocontrol of quantum many-body states. One of the most surprising examples of strong mode coupling is the interaction between apical oxygen phonons and Josephson plasmons in bilayer $\mathrm{YBa}_{2} \mathrm{Cu}_{3} \mathrm{O}_{6+\mathrm{x}}$ superconductors. Experiments by Hu et al. [Nat. Mater. 13, 705 (2014)] and Kaiser et al. [Phys. Rev. B 89, 184516 (2014)] showed that below $T_{c}$, photoexcitation of phonons leads to enhancement and frequency shifts of Josephson plasmon edges, while above $T_{c}$, photoexcited phonons induce plasmon edges even when there are no discernible features in the equilibrium reflectivity spectrum. Recent experiments by von Hoegen et al. (arXiv:1911.08284) also observed parametric generation of Josephson plasmons from photoexcited phonons both below $T_{c}$ and in the pseudogap phase. In this paper, we present a theoretical model of three-wave phonon-plasmon interaction arising from changes of the in-plane superfluid stiffness caused by the apical oxygen motion. Analysis of the parametric instability of plasmons based on this model gives frequencies of the most unstable plasmons that are in agreement with experimental observations. We also discuss how strong parametric excitation of Josephson plasmons can explain pump-induced changes in the terahertz reflectivity of $\mathrm{YBa}_{2} \mathrm{Cu}_{3} \mathrm{O}_{6+\mathrm{x}}$ in the superconducting state, including frequency shifts and sharpening of Josephson plasmon edges, as well as appearance of a new peak around 2 THz. An interesting feature of this model is that overdamped Josephson plasmons do not give any discernible features in reflectivity in equilibrium, but can develop plasmon edges when parametrically excited. We suggest that this mechanism explains photoinduced plasmon edges in the pseudogap phase of $\mathrm{YBa}_{2} \mathrm{Cu}_{3} \mathrm{O}_{6+\mathrm{x}}$.
\end{abstract}

DOI: 10.1103/PhysRevB.102.174505

\section{INTRODUCTION}

\section{A. Motivation}

Photoinduced superconductivity has been one of the most surprising discoveries of pump and probe experiments in solids. By now it has been observed in three different classes of materials: high- $T_{c}$ cuprates [1-7], the buckyball superconductor $\mathrm{K}_{3} \mathrm{C}_{60}$ [8], and organic superconductors such as (BEDT-TTF $)_{2} \mathrm{Cu}\left[\mathrm{N}(\mathrm{CN})_{2}\right] \mathrm{Br}$ [9]. In many of these materials, superconductivity has been induced by tuning the pump pulse to be resonant with one of the infrared (IR) active phonon modes; thus, interaction between the strongly excited phonon modes and the many-body electron system is at the heart of this phenomenon. The primary evidence for transient superconductivity comes from reflectivity measurements, which allow to study optical conductivity $\sigma(\omega)$. Suppression of the real part of the conductivity at low frequencies indicates opening of the quasiparticle gap, and appearance of the $1 / \omega$ tail in the imaginary part suggests the appearance of finite-frequency superfluid stiffness. In the case of high- $T_{c}$ superconductors, and especially $\mathrm{YBa}_{2} \mathrm{Cu}_{3} \mathrm{O}_{6+\mathrm{x}}$, another important indication

*marios_michael@g.harvard.edu of the photoinduced superconducting state has been the appearance of special features in the reflection spectra, called the Josephson plasmon (JP) edge [2,3] (see Sec. IID for a detailed discussion). In equilibrium, JPs are understood to be a consequence of coherent Cooper pair tunneling between the layers, and thus provide a hallmark signature of the superconducting state. Experiments by $\mathrm{Hu}$ et al. [2] and Kaiser et al. [3] showed that following photoexcitation of apical oxygen (AO) phonons in $\mathrm{YBa}_{2} \mathrm{Cu}_{3} \mathrm{O}_{6+\mathrm{x}}$ [Fig. 1(a)], JP edges get strongly enhanced: they sharpen below $T_{c}$ and may appear out of the featureless optical conductivity above $T_{c}$. Moreover, consequences of pumping include the appearance of an additional photoinduced edge below $T_{c}$ and a shift in frequency of JP edges relative to their equilibrium values both below and above $T_{c}$. The appearance of this additional blueshifted lower plasmon edge has been interpreted as indication of the photoinduced enhancement of the interlayer Josephson coupling. Earlier theoretical work aimed at understanding photoinduced superconductivity [10-24] has not been able to explain the dramatic enhancement of JP edges following excitation of the phonons. Another striking phenomenon that has been recently observed in $\mathrm{YBa}_{2} \mathrm{Cu}_{3} \mathrm{O}_{6+\mathrm{x}}$ is the coherent excitation of Josephson plasma wave oscillations by the photoexcited phonons [1]. Intriguingly, such resonant excitation of plasmons has been observed not only below $T_{c}$, but also 


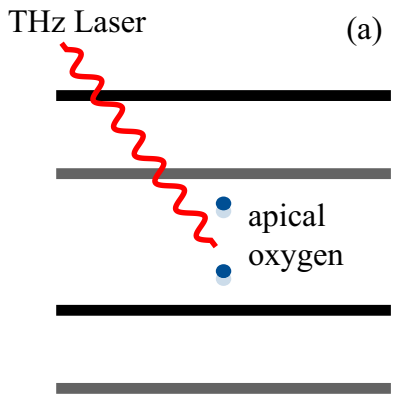

(a)

(b)

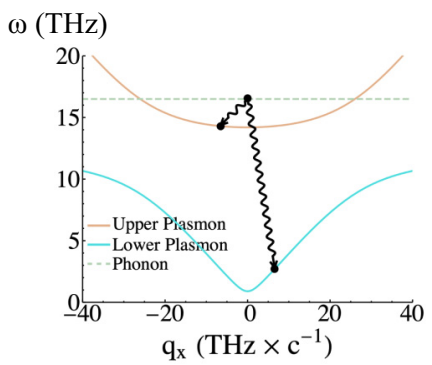

FIG. 1. (a) Schematic representation of the pumping scheme. A laser pulse at frequency $\omega=16.5 \mathrm{THz}$ coherently excites the resonant phonon corresponding to oscillations of the apical oxygen sitting in-between the two layers of the bilayer structure. (b) The orange and blue lines show dispersions of the upper and lower Josephson polaritons as a function of in-plane momentum (see Sec. II for details). The phonon mode at $q_{\mathrm{ph}}=0$ can be resonantly scattered into a pair of JPs with opposite momenta. The resonant scattering condition is $\omega_{\mathrm{ph}}=\omega_{1}(\vec{q})+\omega_{2}(-\vec{q})$. The figure shows the resonant process at $q_{z}=0$ only. There is a full $2 \mathrm{D}$ surface of resonant processes with the parametric instability for different pairs determined by the corresponding matrix elements (see Fig. 9 and Sec. IV for details). The dominant instability occurs for $q_{z}$ near the Brillouin zone boundary.

in the pseudogap phase. The goal of this paper is to provide a unified physical model for the phonon-plasmon interaction in $\mathrm{YBa}_{2} \mathrm{Cu}_{3} \mathrm{O}_{6+\mathrm{x}}$ that explains all these different experimental signatures.

\section{B. Nontechnical summary of the paper}

The starting point of our theoretical study is the experimental finding by von Hoegen et al. [1] that illuminating the superconducting $\mathrm{YBa}_{2} \mathrm{Cu}_{3} \mathrm{O}_{6+\mathrm{x}}$ with light resonant with $\mathrm{AO}$ phonons leads to the appearance of coherent Josephson plasmon excitations. These experiments used second harmonic generation (SHG) measurements at optical frequencies to probe time-dependent breaking of inversion symmetry, which can arise from both phonon displacements and electric currents. From the perspective of $\mathrm{THz}$ dynamics of collective modes, measurements at the optical frequency are essentially instantaneous. Thus, the SHG signal allows measurement of the time dependence of inversion symmetry breaking, which can then be Fourier transformed to determine frequencies of the excited collective modes. Experiments revealed strong signals at frequencies corresponding to lower and upper Josephson plasmons not only below $T_{c}$, but also in the pseudogap phase. This was interpreted as a result of resonant parametric scattering between the photoexcited $\mathrm{AO}$ phonon and two Josephson plasmons in a three-wave mixing process shown in Fig. 1. Further support for this model came from the measured exponential dependence of the induced JP currents on the pump amplitude. In this paper we present a detailed model of phonon-plasmon resonant parametric scattering in $\mathrm{YBa}_{2} \mathrm{Cu}_{3} \mathrm{O}_{6+\mathrm{x}}$, in which phonon-plasmon coupling comes from the modulation of in-plane superfluid density in $\mathrm{Cu}-\mathrm{O}$ layers by apical oxygen phonons. We show that while the parametric resonance condition is satisfied for a range of (a)

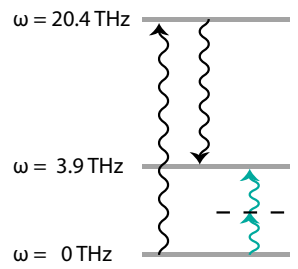

(b)

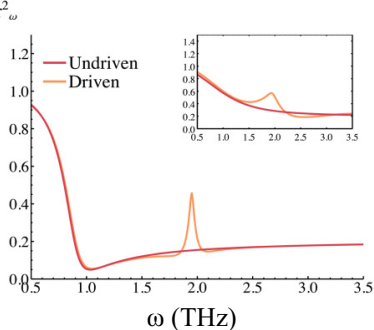

FIG. 2. (a) Schematic representation of the four-wave mixing process renormalizing the reflection coefficient. In this four-wave mixing process the sum of a pair of plasmons (green) adds up to the subtraction of neighboring phonon frequencies (black). (b) Reflection coefficient with and without driving. Inset: emergence of a photoinduced edge from a featureless background in the case of strongly dissipative plasmons.

upper and lower Josephson plasmon pairs, the most unstable pair has frequencies of 2.2 and $14.3 \mathrm{THz}$. These frequencies are in good agreement with the experimentally measured values [1].

The second goal of this paper is to show that nonlinear couplings between collective modes, including both phononplasmon and plasmon-plasmon interactions, provide a natural explanation for previously observed changes in the terahertz reflectivity. To understand these phenomena, we need to broaden the discussion of parametric resonant scattering processes to include second-order processes, in which phonon-induced 2.2 and $14.3 \mathrm{THz}$ plasmons themselves become a source of parametric driving. While there are many possibilities for higher-order parametric instabilities, we can identify the ones that are most important for understanding the renormalization of optical reflectivity from considerations of energy and momentum conservation. For example, we find that reflectivity close to the lower plasmon edge is renormalized either by a four-wave mixing of two neighboring phonons and two lower Josephson plasmons, as shown in Fig. 3(a), or a four-wave mixing between four lower plasmons as shown in Fig. 11 (discussed in Sec. V). Both mechanisms give rise to similar features in reflectivity, shown in Fig. 2(b).

The main focus of our paper is understanding pumpinduced changes in the terahertz reflectivity of $\mathrm{YBa}_{2} \mathrm{Cu}_{3} \mathrm{O}_{6+\mathrm{x}}$ in the superconducting state. We demonstrate that parametric driving can explain renormalization of JP energies, sharpening of the edges, and appearance of the new edge around $2 \mathrm{THz}$. We also provide a qualitative discussion of the pseudogap regime, which we envision as having short-ranged superconducting correlations [25-34]. Earlier analysis of Josephson plasmons in the vortex liquid state of high- $T_{c}$ cuprates by Bulaevskii and Koshelev [35] has already established that finite correlations between neighboring layers are sufficient for observing plasmons as collective polaritonic modes. The main consequence of the absence of long-range superconducting order is enhanced broadening of the plasmon resonance. Furthermore, in the pseudogap regime, one expects higher damping of plasmons. This damping comes from an increase in the real part of optical conductivity at the plasmon frequency; in the superconducting state below the frequency of the quasiparticle continuum, the real part of conductivity of 
$d$-wave superconductors is strongly suppressed [36-38]. In this spirit we treat Josephson plasmons in the pseudogap regime as overdamped excitations that undergo parametric driving following photoexcitation. We demonstrate that resonant parametric driving can explain the emergence of the lower JP edge out of the featureless continuum in reflectivity data; this is the most striking feature of the "light-induced superconductivity" in the pseudogap regime of $\mathrm{YBa}_{2} \mathrm{Cu}_{3} \mathrm{O}_{6+\mathrm{x}}$.

\section{Organization of the paper}

Josephson plasmons and terahertz reflectivity in equilibrium. We begin Sec. II by presenting a microscopic theory of JPs in bilayer superconductors, such as $\mathrm{YBa}_{2} \mathrm{Cu}_{3} \mathrm{O}_{6+\mathrm{x}}$. Our analysis is based on combining hydrodynamic equations of the superconducting order parameter with Maxwell equations for electric and magnetic fields. This approach is equivalent to the linearized version of the three-dimensional sine-Gordontype equation of electrodynamics of layered superconductors obtained in Refs. [39-42]. In contrast to these papers we retain electric and magnetic fields as explicit degrees of freedom, in order to facilitate identification of eigenmodes, analysis of resonant scattering processes, and computation of the frequency-dependent reflectivity. We conclude the discussion of the equilibrium properties of plasmons by presenting a simple analytical model for calculating reflectivity in a bilayer superconductor and show that results of this analysis are in good agreement with experiments. In Sec. III we introduce $\mathrm{AO}$ phonon modes and review symmetry constraints on their nonlinear coupling to Josephson plasmons. We suggest that the microscopic origin of phonon-plasmon interaction is modulation of the in-plane superfluid stiffness by the apical oxygen displacements, coming from modifications either in the density or the effective mass of the charge carriers. It is useful to note that phonon-plasmon coupling can not be captured using the approach of Refs. [13,43-46], which treats a layered superconductor as a chain of Josephson junctions [13,40,43-47] stacked along the $c$ axis, thus neglecting the in-plane superfluid currents. Due to symmetry constraints three-wave phonon-plasmon coupling only takes place for finite in-plane momentum of plasmons.

Parametric instabilities and terahertz reflectivity in a driven state. Section IV presents analysis of the parametric instability in which one photoexcited phonon generates a pair of Josephson polaritons that have opposite momenta and energies that add up to the phonon frequency (see Fig. 1). The parametric resonance condition does not select a unique pair of plasmons, but is satisfied for JPs on a two-dimensional (2D) surface in momentum space. We calculate the growth rate for all resonant pairs and find that the most unstable modes have frequencies 2.2 and $14.3 \mathrm{THz}$, in agreement with the second harmonic generation experiments [1]. In Sec. V we extend analysis of the terahertz reflectivity of bilayer superconductors to driven systems using the perspective of Floquet medium [48,49]. In this discussion sources of the drive can be either the original photoexcited phonons, or the unstable plasmon modes identified in Sec. IV (see Ref. [50] for a discussion on considering the incoming electric field from the pump field directly as the source of the drive). For each frequency window considered, dominant contributions come from processes that are closest to parametric resonance and consistent with symmetry selection rules. In the frequency region close to the lower JP edge, the above considerations suggest two mechanisms that can lead to renormalization of the reflection coefficient: (i) a four-wave mixing process that involves two different AO phonons and two lower JPs, (ii) a four-wave mixing process between the lower JPs, in which two plasmons have been generated by the phonon decay. Both modulation mechanisms are expected to result in a similar change of the reflection coefficient, with the strongest feature being a new peak at the frequency around $2 \mathrm{THz}$. Reflection at frequencies close to the upper JP edge is modified by the four-plasmon interaction, where two plasmons have been generated in the decay of the photoexcited phonon. On a technical level, theoretical tools developed in this section represent a generalization of previous analysis of reflectivity from a Floquet medium [48,49] to the case of multiple plasmon-polariton bands corresponding to different transmission and reflection channels for light within the material. We decompose electromagnetic fields and matter excitations into eigenstates corresponding to different plasmon-polariton bands and project light reflection problem to the relevant eigenstate subspace for the frequency window of interest. Within that subspace we include only resonant time-dependent processes, equivalent to a first-order Floquet degenerate perturbation scheme [51]. In practice, this means keeping only signal and idler frequency components for the different transmission channels that we consider [52]. Our analysis does not include depletion of the driving modes and therefore only captures short-time dynamics of the pump and probe experiments. In Sec. V we also demonstrate that in systems with overdoped JPs, which do not show plasma edges in equilibrium, parametric driving can lead to the appearance of the edge. We suggest that this can explain light-induced superconductivity in the pseudogap phase of $\mathrm{YBa}_{2} \mathrm{Cu}_{3} \mathrm{O}_{6+\mathrm{x}}$. In $\mathrm{Sec}$. VI, we summarize our results and review open problems.

\section{THEORETICAL ANALYSIS OF JOSEPHSON PLASMONS IN BILAYER SUPERCONDUCTORS}

We analyze dynamics of a bilayer system such as $\mathrm{YBa}_{2} \mathrm{Cu}_{3} \mathrm{O}_{6+\mathrm{x}}$ by developing a microscopic description based on phase and density fluctuations in each superconducting layer $\left\{\delta \rho_{i, \lambda}, \phi_{i, \lambda}\right\}$, coupled to the four-component vector potential $\left(V_{\lambda, i}(\vec{x}), A_{\lambda, i, z}(\vec{x}) \vec{A}_{\lambda, i, \vec{x}}(\vec{x})\right)$ through Maxwell's equations. Here, $i$ corresponds to the index of the unit cell along the $c$ axis, $\lambda=1,2$ labels the number of the layer inside the unit cell, and $\vec{x}$ is the in-plane coordinate, which we will omit in the equations below for brevity. While the in-plane components of the vector potential $\vec{A}_{\lambda, i, \vec{x}}(\vec{x})$ are defined within the corresponding layers, $A_{\lambda, i, z}(\vec{x})$ is defined to be on the links between layers starting on layer $\{\lambda, i\}$ as shown in Fig. 3. Our analysis focuses on density and current fluctuations since we expect super flow currents to dominate at frequencies below $2 \Delta$. In Sec. II D, we add dissipation phenomenologically to include effects of quasiparticle excitations. Equations of motion are given by hydrodynamic equations discretized along the $c$ axis. Our treatment expands on ideas developed in previous works treating $\mathrm{YBa}_{2} \mathrm{Cu}_{3} \mathrm{O}_{6+\mathrm{x}}$ as a one-dimensional (1D) chain of Josephson junctions [13,40,43-46] to include in-plane 


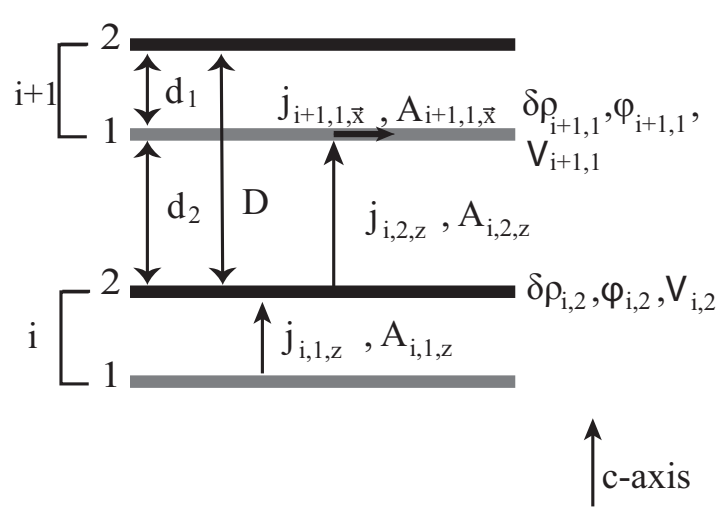

FIG. 3. Schematic view of the $\mathrm{YBa}_{2} \mathrm{Cu}_{3} \mathrm{O}_{6+x}$ material. $\mathrm{YBa}_{2} \mathrm{Cu}_{3} \mathrm{O}_{6+\mathrm{x}}$ consists of a bilayer array of superconducting $\mathrm{CuO}_{2}$ layers. Each layer is described by the density and phase fluctuations of the superconductor. Electric fields and currents along the $c$ axis live in the space between the layers while in-plane electric fields and currents live inside the layer.

currents in a consistent way. Dynamics of density fluctuations is given by the continuity equation,

$$
\partial_{t} \delta \rho_{\lambda, i}+\partial_{x} j_{\lambda, i, x}+\Delta_{z} j_{\lambda, i, z}=0 .
$$

In linearized hydrodynamics the supercurrents are given by [53]

$$
\begin{aligned}
& j_{\lambda, i, x}=\Lambda_{s}\left(\partial_{x} \phi_{\lambda, i}-e^{*} A_{\lambda, i, x}\right), \\
& j_{\lambda, i, z}=j_{c \lambda} d_{\lambda}^{2}\left(\Delta_{z} \phi_{\lambda, i}-e^{*} A_{\lambda, i, z}\right) .
\end{aligned}
$$

Here, $\vec{x}$ denotes the in-plane $x, y$ components and $z$ axis denotes the $c$ axis of the crystal, coupling to the vector potential is given by the Cooper pair charge $e^{*}=2 e$ and we work in units where $\hbar=1$ for the rest of the paper. The in-plane components of the superfluid current are defined within individual layers and have continuous gradients. The $z$ component of the current is defined as the Josephson current between adjacent layers and has a lattice gradient which corresponds to the phase difference between adjacent layers:

$$
\Delta_{z} \phi_{\lambda, i}=\left\{\begin{array}{c}
\left(\phi_{2, i}-\phi_{1, i}\right) / d_{1} \text { for } \lambda=1, \\
\left(\phi_{1, i+1}-\phi_{2, i}\right) / d_{2} \text { for } \lambda=2 .
\end{array}\right.
$$

In the continuity equation (1), the lattice gradient of $j_{\lambda, i, z}$ (defined on links) corresponds to the difference of currents flowing in and out of the $\{\lambda, i\}$ layer and is given by [53]

$$
\begin{aligned}
& \Delta_{z} j_{\lambda, i, z}=\left\{\begin{array}{l}
\frac{j_{1, i, z}}{d_{1}}-\frac{j_{2, i-1, z}}{d_{2}} \text { for } \lambda=1, \\
\frac{j_{2, i, z}}{d_{2}}-\frac{j_{1, i, z}}{d_{1}} \text { for } \lambda=2 .
\end{array}\right. \\
& H_{\mathrm{EM}}=\int d^{2} x \sum_{i, \lambda} \frac{c^{2}}{2 \epsilon} P_{V, \lambda, i}^{2}+\frac{\epsilon}{2}\left(\left(\partial_{x} V_{\lambda, i}\right)^{2}+\left(\Delta_{z} V_{\lambda, i}\right)^{2}\right)+\frac{1}{2 \epsilon} P_{A_{x}, \lambda, i}^{2}+\frac{\epsilon c^{2}}{2}\left(\left(\partial_{x} A_{\lambda, i, x}\right)^{2}+\left(\Delta_{z} A_{\lambda, i, x}\right)^{2}\right) \\
& +\frac{1}{2 \epsilon} \frac{P_{A_{z}, \lambda, i}^{2}}{2}+\frac{\epsilon c^{2}}{2}\left(\left(\partial_{x} A_{\lambda, i, z}\right)^{2}+\left(\Delta_{z} A_{\lambda, i, z}\right)^{2}\right),
\end{aligned}
$$

where the fields $\left\{P_{V, \lambda, i}, P_{A_{x}, \lambda, i}, P_{A_{z}, \lambda, i}\right\}$ have been introduced and correspond to the conjugate momenta of the components of the electrostatic and vector potentials. Canonically conjugate pairs $\rho$ and $\phi, V$ and $P_{V}, \vec{A}$ and $P_{\vec{A}}$
Coefficient $\Lambda_{s}$ is related to the in-plane London penetration length as $\Lambda_{s}=\frac{\epsilon c^{2}}{\lambda_{L}^{2}\left(e^{*}\right)^{2}}$, where $\epsilon=\epsilon_{r} \epsilon$ the permittivity of the material. Physically, it corresponds to the intralayer superfluid stiffness and is proportional to the condensate density $\Lambda_{s \lambda} \propto$ $\rho_{\lambda}$. In linear analysis of collective modes we can set $\Lambda_{s \lambda}$ to be equal to their equilibrium values since they multiply superfluid velocities $\vec{v}_{\lambda, i}=\partial_{\vec{x}} \phi_{\lambda, i}-e^{*} A_{\lambda, i, \vec{x}}$, which are already first order in fluctuations. This is why we omitted the layer index for $\Lambda_{s}$ in Eq. (2). Coefficients $\left\{j_{c, \lambda}\right\}$ correspond to interlayer Josephson tunneling couplings and obey $j_{c, \lambda} \propto \sqrt{\rho_{1} \rho_{2}}$. In linearized hydrodynamics, we take $j_{c, \lambda}$ to be equal to their equilibrium value and neglect corrections due to $\delta \rho_{\lambda}$. Phase dynamics are given through the Josephson relation

$$
\partial_{t} \phi_{\lambda, i}=-\gamma \delta \rho_{\lambda, i}-e^{*} V_{\lambda, i} .
$$

In Eq. (5), $\gamma$ arises from a finite electron compressibility [43] and can be linked to the Thomas-Fermi length by $\gamma=\frac{\lambda_{\mathrm{TF}}^{2}\left(e^{*}\right)^{2}}{\epsilon}$.

To consistently couple to electromagnetism, one needs to use discrete QED along the $c$ axis and continuous QED in plane. In the Lorenz gauge, the Maxwell's equations obtain the familiar form

$$
\begin{aligned}
\left(\frac{1}{c^{2}} \partial_{t}^{2}-\partial_{x}^{2}-\Delta_{z}^{2}\right) V_{\lambda, i} & =\frac{\left(e^{*}\right)}{\epsilon} \delta \rho_{\lambda, i}, \\
\left(\frac{1}{c^{2}} \partial_{t}^{2}-\partial_{x}^{2}-\Delta_{z}^{2}\right) A_{\lambda, i, x} & =\frac{1}{c^{2}} \frac{\left(e^{*}\right)}{\epsilon} j_{\lambda, i, x}, \\
\left(\frac{1}{c^{2}} \partial_{t}^{2}-\partial_{x}^{2}-\Delta_{z}^{2}\right) A_{\lambda, i, z} & =\frac{1}{c^{2}} \frac{\left(e^{*}\right)}{\epsilon} j_{\lambda, i, z},
\end{aligned}
$$

where along the $c$ axis, lattice gradients are used and the magnetic permeability $\mu=\mu_{r} \mu_{0}$ is included through the speed of light in the material $c=\frac{1}{\sqrt{\mu \epsilon}}$. The discretized Lorenz gauge condition is given by

$$
\frac{1}{c^{2}} \partial_{t} V_{\lambda, i}+\partial_{x} A_{\lambda, i, x}+\Delta_{z} A_{\lambda, i, z}(x, t)=0 .
$$

With the constraint in Eq. (7), the above equations of motion (1)-(6) can be compactly encoded by the Hamiltonian

$$
\begin{aligned}
H= & \int d^{2} x \sum_{i, \lambda}\left\{\frac{\gamma}{2} \delta \rho_{\lambda, i}^{2}+\frac{1}{2 \Lambda_{s}} j_{\lambda, i, x}^{2}\right. \\
& \left.+\frac{1}{2 j_{c, \lambda} d_{\lambda}^{2}} j_{\lambda, i, z}^{2}+e^{*} \delta \rho_{\lambda, i} V_{\lambda, i}+H_{\mathrm{EM}}\right\},
\end{aligned}
$$

where $H_{\mathrm{EM}}$ gives rise to Maxwell's equations of motion. In obey canonical commutations relations, i.e., $\left[\rho(\vec{x})_{i}, \phi_{j}\left(\vec{x}^{\prime}\right)\right]=$ $i \delta\left(\vec{x}-\vec{x}^{\prime}\right) \delta_{i, j}$. Equations of motion for the operators are recovered through Heisenberg equations of motion $\partial_{t} \hat{O}=$ $i[\hat{H}, \hat{O}]$. 
The advantage of using the Lorenz gauge is evident from the fact that different gauge fields decouple from each other [Eq. (6)]. The price we pay is that, unlike the Coulomb gauge, the Hamiltonian in Eq. (9) has additional unphysical degrees of freedom that can be removed through the gauge condition in Eq. (7).

\section{A. Quantization in the Lorenz gauge}

Quantization in the Lorenz gauge follows a method introduced by Gupta and Bleuler $[54,55]$. The Hamiltonian is quantized by treating each gauge field as a scalar. This introduces unphysical polarizations, namely, a longitudinal and a scalar polarization. These are removed by defining the physical Hilbert space as the set of states that satisfy the Lorenz gauge condition [Eq. (7)]. While the literature typically focuses on QED in free space, we discuss in this section how this idea is applied for QED coupled to matter in the particular example presented in this paper.

Starting from the equations of motion (1)-(6), dispersion relations of elementary excitations of the system (plasmons) are found by looking for plane-wave solutions:

$$
\delta \rho_{\lambda, i}(\vec{x}, t)=\delta \rho_{\lambda}\left(q_{x}, q_{z}, \omega\right) e^{i q_{\vec{x}} \vec{x}+i q_{z} D i-i \omega t} .
$$

In the Hamiltonian description of the equations of motion where the momenta of the gauge fields $\left\{P_{V}, P_{A_{x}}, P_{A_{z}}\right\}$ are included, the equations of motion become first order in the time derivatives and take the form

$$
i \partial_{t} \vec{v}=M \cdot \vec{v},
$$

where $\underline{v}$ is the vector containing the variables in either layer of $\mathrm{YBa}_{2} \mathrm{Cu}_{3} \mathrm{O}_{6+\mathrm{x}}, \quad \vec{v}=\left\{\rho_{\lambda}, V_{\lambda}, A_{\lambda, x}, A_{\lambda, z}, \phi_{\lambda}, P_{V, \lambda}, P_{A_{x}, \lambda}, P_{A_{z}, \lambda}\right\}$ and $\lambda=\{1,2\} . M$ is a $16 \times 16$ matrix coupling the different variables together and contains gradient operators. Substituting the plane-wave ansatz this reduces to a $16 \times 16$ eigenvalue problem of the type

$$
\omega \underline{v}(\vec{q}, \omega)=M(\vec{q}) \cdot \underline{v}(\vec{q}, \omega) .
$$

The dispersion of elementary excitations is given by solving the corresponding secular equation

$$
\chi(\omega)=\operatorname{det}(\underline{\underline{M}}-\omega)=0 .
$$

In Eq. (13), $\chi(\omega)$ is the characteristic polynomial of the equations of motion whose solution contains both physical and unphysical energy states. However, the gauge condition (7) and continuity equation (8) guarantee that the unphysical degrees of freedom are decoupled from the matter fields $\{\rho, \phi\}$. This decoupling of matter fields and unphysical degrees of freedom holds for a generic system and not just this example. As a result, the characteristic polynomial factorizes into physical and unphysical contributions $\chi(\omega)=\chi_{\text {phys }}(\omega) \chi_{\text {unphys }}(\omega)$. Therefore, coupling to matter provides a particularly simple recipe for constructing the physical Hillbert space by ignoring $\chi_{\text {unphys }}(\omega)$ and solving only for

$$
\chi_{\text {phys }}(\omega)=0 .
$$

This procedure amounts to explicitly removing the unphysical eigenstates from the theory. Finally, in order to restrict the theory to the physical Hilbert space, variables are expressed as a linear combination of the eigenvectors corresponding to the physical eigenstates only:

$$
\left(\begin{array}{c}
\delta \rho_{\lambda}(q) \\
\vdots \\
\phi_{\lambda}(q) \\
\vdots
\end{array}\right)=\left(\begin{array}{cccc}
v_{1, q}^{\delta \rho_{\lambda}} & \cdots & \left(v_{1, q}^{\delta \rho_{\lambda}}\right)^{*} & \cdots \\
\vdots & & \vdots & \\
v_{1, q}^{\phi_{\lambda}} & & \left(v_{1, q}^{\phi_{\lambda}}\right)^{*} & \\
\vdots & & \vdots &
\end{array}\right)\left(\begin{array}{c}
b_{1} \\
\vdots \\
b_{1}^{*} \\
\vdots
\end{array}\right) .
$$

Quantization of the theory is completed by promoting the coefficients of eigenvectors of the secular equation to creation and annihilation operators with bosonic commutation relations:

$$
\left[\hat{b}_{i}, \hat{b}_{j^{\prime}}^{\dagger}\right]=\delta_{i, j} .
$$

This choice fixes the normalization constant of the eigenvectors as shown and completes our construction as outlined in Appendix A. Details of the mathematical structure of the diagonalization scheme can be found in Ref. [56] where one can prove that for a real spectrum, the eigenvectors form an orthonormal basis of the physical system. Nonlinearities and other terms in the Hamiltonian can be added without coupling to unphysical degrees of freedom as long as the operators are expanded only as a linear combination of the physical creation and annihilation operators.

\section{B. Plasmons}

Solving the equations of motion in the previous section, we find three energy scales at zero momentum which correspond to three distinct plasmons (see Appendix B). The intralayer superfluid stiffness $\Lambda_{s}$ sets the energy of the in-plane plasmon which corresponds to in-plane supercurrent oscillations:

$$
\omega_{\text {in plane }}=\sqrt{\Lambda_{s} \frac{\left(e^{*}\right)^{2}}{\epsilon}} .
$$

The other two energy scales are associated with the two interlayer Josephson tunneling couplings $\left\{j_{c, \lambda}\right\}$ and set the energy gap for the two JPs,

$$
\omega_{\{1,2\}} \approx \sqrt{j_{c,\{1,2\}} d_{1,2}^{2} \frac{\left(e^{*}\right)^{2}}{\epsilon}},
$$

where $\left\{\gamma, \epsilon, \frac{d_{1}}{d_{2}}\right\}$ couple the two plasmons together and offer corrections to this intuition. Due to the large separation of scale between $\Lambda_{s}$ and $j_{c, \lambda}$, the in-plane plasmon corresponds to a high-energy excitation decoupled from the JPs that are low in energy $\left(\omega_{\text {upper JP }}=14.2 \mathrm{THz}, \omega_{\text {lower JP }}=0.9 \mathrm{THz}\right)$. The JPs are the relevant degrees of freedom that are accessible in the experimental setup. The dispersion relation at small momenta is given in Figs. 4 and 5.

Previous studies $[13,40,43-46]$ used the fact that the inplane plasmon is very high in energy to essentially ignore the effects of the in-plane kinetic energy at very small momenta $q_{x} \ll \frac{\omega_{\text {in plane }}}{c}$. While this may be sufficient to calculate optical properties of the system, we show in the next section that the phonon coupling to the in-plane kinetic energy dominates dynamics in the pump and probe experiments. Moreover, the prediction that the lower plasmon saturates at large $q_{x}$ is 


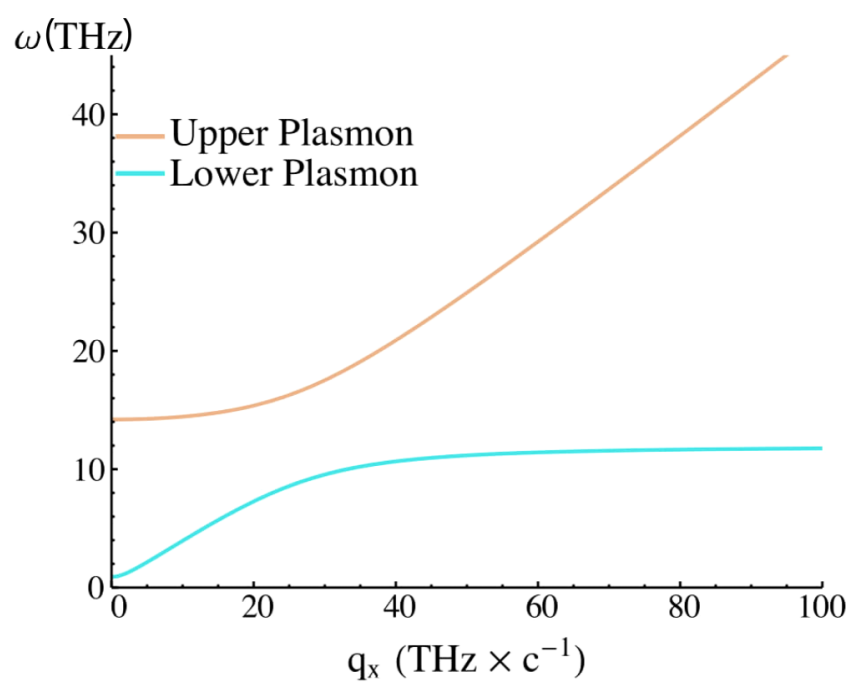

FIG. 4. Dispersion relation of two lower plasmons. For small wave vectors $q_{x} \ll \frac{\sqrt{\Lambda_{s}}}{c}$, one can ignore the presence of the in-plane plasmon at equilibrium.

approximately true as shown by Fig. 4, but fails in the nonrelativistic limit $q_{x} \gg \frac{\omega_{\text {inplane }}}{c}$, where the in-plane kinetic energy dominates and the dispersion increases linearly with the inplane superfluid velocity $v_{s} \approx \sqrt{\Lambda_{s} \gamma}$ (shown in Fig. 17). Crucially, the presented formalism allows exploration of the full $\left(q_{\vec{x}}, q_{z}\right)$ plane as shown in Fig. 5, which is necessary in order to find the most unstable mode during parametric driving (see Sec. IV).

Eigenvectors of JPs correspond to the low-energy eigenvectors of the equations of motion (11) and allow us to project dynamics to the low-energy subspace using the recipe described in Appendix A. In particular, this method is used in Sec. III to derive an effective phonon-plasmon Hamiltonian starting from a physical coupling between phonons and superfluid currents. The same approach can be used to reduce nonlinear corrections to the hydrodynamic equations of motion to a theory of interacting plasmons. Moreover, eigenvectors encode physical properties of JPs such as their overlap with electromagnetic fields, which are important in calculating reflectivity as outlined in Sec. II D.

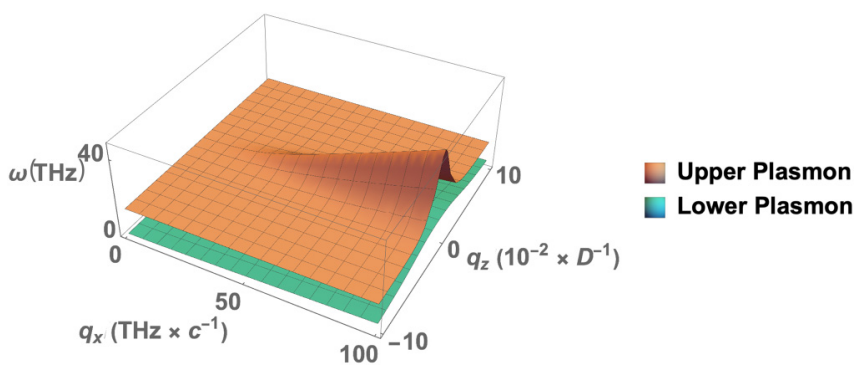

FIG. 5. Dispersion relation of two lower plasmons in the $\left\{q_{x}, q_{z}\right\}$ plane. At $q_{z}=0$, the upper plasmon is strongly coupled to the photon and its dispersion gradient approaches the speed of light, while away from $q_{z}=0$ the energy decreases.

\section{Reflection symmetries and collective modes in $\mathrm{YBa}_{2} \mathrm{Cu}_{3} \mathrm{O}_{6+\mathrm{x}}$}

In the literature the phonon modes and other collective excitations are usually grouped in a convenient way with respect to their reflection symmetry in the plane parallel to the crystallographic $a-b$ plane. Here we comment on the symmetry of the JPs and the relevant phonon modes, and clarify what this implies for possible interactions between different modes. The Hamiltonian defined in Eq. (8) obeys the reflection symmetry in the plane parallel to the crystallographic $a-b$ plane, defined as

$$
P \delta \rho_{\{1,2\}, i}(x, t) P^{\dagger}=\delta \rho_{\{2,1\},-i}(x, t)
$$

with similar relations for other operators $j_{\lambda, i, \vec{x}}, V_{\lambda, i}$, etc., while for vectors along the $z$ axis the reflection symmetry transformation becomes

$$
\begin{aligned}
& P A_{1, i, z} P^{\dagger}=-A_{1,-i, z}, \\
& P A_{2, i, z} P^{\dagger}=-A_{1,-i-1, z} .
\end{aligned}
$$

This implies that the JP eigenvectors can be written as eigenstates of the reflection operator. Written in the momentum basis, only JPs at special points in the Brillouin zone have definite reflection symmetry. Here we report that at $q_{z}=0$ for arbitrary $q_{x}$, JPs are odd under reflection while at the edge of the Brillouin zone and at $q_{z}=\frac{\pi}{D}$ and arbitrary $q_{x}$ the upper plasmon is odd while the lower plasmon is even under reflection (shown schematically in Fig. 6). Away from these special points, linear combinations of modes with opposite momenta can be combined to produce either parity. Similarly to the electronic sector, there are two $\mathrm{AO}$ optical phonons of interest at zero momentum in $\mathrm{YBa}_{2} \mathrm{Cu}_{3} \mathrm{O}_{6+\mathrm{x}}$, one at $16.5 \mathrm{THz}$ $\left(Q_{1}\right)$ and one at $20.4 \mathrm{THz}\left(Q_{2}\right)$ (which are resonant with the probe). These photoexcited phonons are both odd under reflection symmetry. This implies that a three-wave mixing between JP modes at $q_{z}=0$ and a phonon is forbidden while it is allowed for finite $q_{z}$ and in particular at $q_{z}=\frac{\pi}{D}$. As a result, a three-wave mixing process of the type shown in Fig. 1 involving a phonon-driven parametric instability of a plasmon pair is expected to necessarily excite plasmons at finite momentum. Such excitations can be measured in second harmonic generation experiments and the dominant pair is computed in Sec. IV. On the other hand, when discussing reflectivity experiments, the incoming probe beam couples most strongly with JPs at $q_{z}=0$. From symmetry arguments these modes can participate in four-wave mixing processes involving two phonons and two lower plasmons at $q_{z}=0$ as shown schematically in Fig. 2(a). The photoinduced reflectivity spectrum arising from such mixing is computed in Sec. V.

The material has in fact mirror symmetries in all three axes usually denoted by “ $\mathrm{mmm}$." The presence of a Josephson plasmon breaks the mirror along the $z$ direction while finite in-plane momentum breaks the mirror along the direction parallel to the in-plane momentum, reducing the symmetry from $m m m \rightarrow m$.

\section{Static reflectivity}

We conclude our discussion on the equilibrium properties of the system by computing the equilibrium reflectivity predicted by this theory. We develop a simplified analytical 

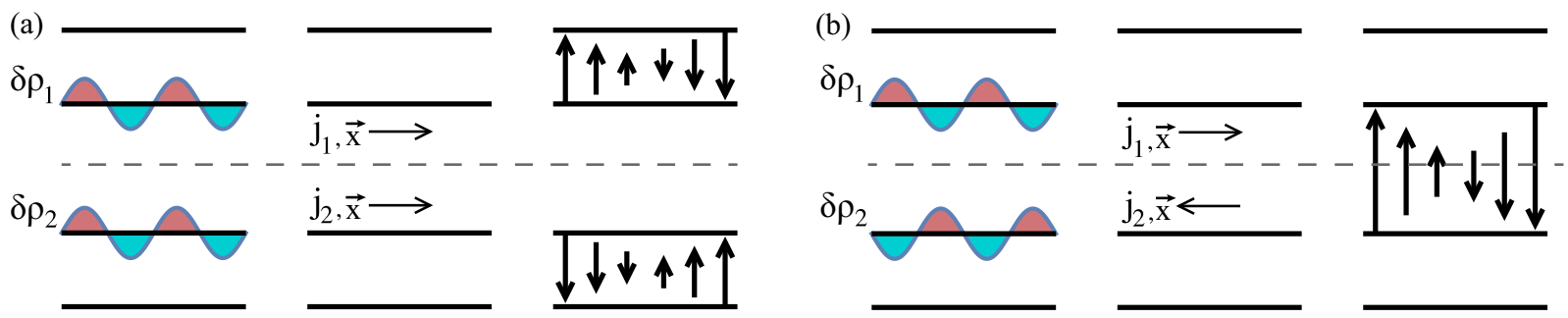

FIG. 6. Schematic representation of the pair of unstable plasmon modes $q_{z}=\frac{\pi}{D}$ and $q_{x}$ finite. The dashed line corresponds to the crystallographic $a-b$ plane through which the reflection symmetry is defined. At this high-symmetry point (a) the lower plasmon is even under while (b) the upper plasmon is odd.

framework for solving the Fresnel light reflection problem for a bilayer superconducting material such as $\mathrm{YBa}_{2} \mathrm{Cu}_{3} \mathrm{O}_{6+\mathrm{x}}$, and show that it can capture results obtained in reflectivity experiments. We will only discuss the case of normal incidence with the electric field polarized along the $c$ axis of the crystal, as shown schematically in Fig. 7. We take the incident wave (signal) to be given by

$$
\underline{E}_{\text {inc }}=\underline{\hat{z}} E_{0} e^{i q_{x} x-i \omega_{s} t} .
$$

Correspondingly, the $B$ field lies in the $y$ direction and is given by

$$
\underline{B}_{\mathrm{inc}}=-\hat{\hat{y}} \frac{E_{0}}{c_{\mathrm{vac}}} e^{i q_{x} x-i \omega_{s} t},
$$

where $c_{\mathrm{vac}}=\frac{1}{\sqrt{\epsilon_{0} \mu_{0}}}$. The boundary conditions between air and material are given by the requirement that both the $B$ field and $E$ field are continuous across the boundary at every point along the interface, $x=0$ :

$$
\begin{aligned}
& E_{z, \text { inc }}(z, t)+E_{z, r}(z, t)=E_{z, t}(z, t), \\
& B_{y, \text { inc }}(z, t)+B_{y, r}(z, t)=B_{y, t}(z, t),
\end{aligned}
$$

where $\left\{E_{z, r}, B_{y, r}\right\}$ are the amplitudes of the reflected wave and $\left\{E_{z, t}, B_{y, t}\right\}$ the transmitted wave. Due to the lattice periodic structure of the material, the equations of motion couple the $q_{z}=0$ contribution of the transmitted field to all higher harmonics $q_{z}=\frac{2 \pi}{D} n$, where $n$ is an integer. In the spirit of the simplified model presented in Sec. II, we represent the transmitted wave as piecewise constant and characterized by

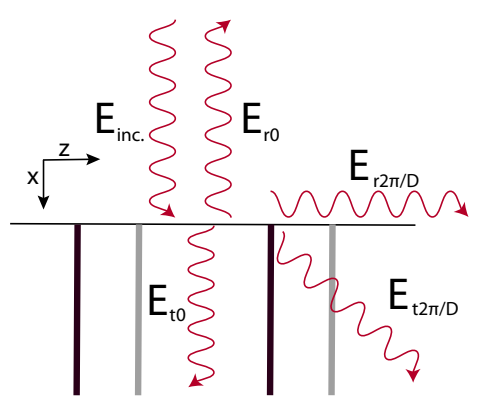

FIG. 7. Schematic representation of reflection at normal incidents. Electric field is assumed to be along the $z$ axis. Due to the periodic structure of the material, light can be reflected and transmitted at momenta $q_{z}=\frac{2 \pi n}{D}$. the interlayer fields $\left\{E_{z, 1}, E_{z, 2}\right\}$. The momentum components of the electric field inside the material are given as

$$
\begin{aligned}
E_{t, q_{z}=0} & =\frac{E_{z, 1} d_{1}+E_{z, 2} d_{2}}{D}, \\
E_{t, q_{z}=\frac{2 \pi n}{D}} & =\left(E_{z, 1}-E_{z, 2}\right) \frac{\left(e^{-i \frac{2 \pi n}{D}}-1\right)}{D}
\end{aligned}
$$

and similarly for $B_{y, t, q_{z}}$. To satisfy the boundary conditions, light is also reflected as an evanescent wave at all these harmonics according to the boundary conditions

$$
\begin{aligned}
E_{z, r, q_{z}} & =r_{q_{z}} E_{0}, \\
B_{y, r, q_{z}} & =\frac{\sqrt{\frac{\omega_{s}^{2}}{c_{\mathrm{vac}}^{2}}-q_{z}^{2}}}{\omega_{s}} r_{q_{z}} E_{0} .
\end{aligned}
$$

Note that due to the simplified piecewise structure of the transmitted fields, the boundary conditions need to be truncated to the first reciprocal lattice vector $q_{z}=\frac{2 \pi}{D}$. In order to take higher harmonics into account, we would need to include high-energy bands, that are sensitive to the more detailed spatial structure of electric and magnetic fields between the layers. Contributions from such high-energy bands are expected to be negligible at frequencies that we consider and are therefore omitted. The boundary conditions are then given by

$$
\begin{aligned}
& E_{0}\left(1+r_{0}\right)=E_{t, 0}, \\
&-\frac{E_{0}}{c_{\mathrm{vac}}}\left(1-r_{0}\right)=B_{t, 0}, \\
& E_{0} r_{\frac{2 \pi}{D}}=E_{t, \frac{2 \pi}{D},} \\
& \frac{i \sqrt{\left(\frac{2 \pi}{D}\right)^{2}-\frac{\omega_{s}^{2}}{c_{\mathrm{vac}}^{2}}}}{\omega_{s}} E_{0} r_{\frac{2 \pi}{D}}=B_{t, \frac{2 \pi}{D},}
\end{aligned}
$$

where in the last line we note that $\frac{2 \pi}{D}>\frac{\omega_{s}}{c_{\text {vac }}}$ for the frequencies of interest and the reflected wave will be evanescent. The transmitted electric and magnetic fields inside the material are related to each other through the equations of motion. As described in Sec. II A, the equations of motion can be cast in the form

$$
i \partial_{t} \vec{v}(\vec{q})=M(\vec{q}) \vec{v}(\vec{q}),
$$

where $\underline{v}$ is a vector containing as its elements the electromagnetic four-component vector potential and density and phase fluctuations of the superconducting state. $M$ is a matrix containing gradient operators. Eigenmodes computed in Sec. II B 
for the bulk $\mathrm{YBa}_{2} \mathrm{Cu}_{3} \mathrm{O}_{6+\mathrm{x}}$ material correspond to solutions of the equations of motion of the type $v=v(q, \omega) e^{i q x-i \omega t}$. When analyzing collective modes in the bulk, for each value of $q$, the dispersion relation of the plasmons is found by computing $\omega$. To solve the boundary problem in reflectivity experiments, the problem is reversed: for each frequency $\omega=\omega_{s}$ specified by the incident electromagnetic wave one computes the wave vectors $q$ oscillating at that frequency. A challenging aspect of the light reflection problem is that one should look for solutions in the full $q$-complex plane, where imaginary values of $q$ are now allowed and correspond to evanescent waves, localized near the boundary. For each solution, the corresponding eigenvector encodes the relative amplitude between electric and magnetic fields for a given transmission channel. This eigenvector is found by the kernel of the matrix $\omega-M(q)$, i.e.,

$$
[\omega-M(q)] v_{q, \omega}=0 .
$$

The electromagnetic 4-vector is expanded in the different transmission channels with complex wave vectors $q=p+i \kappa$ as follows:

$$
V_{\lambda, i}=E_{0}\left(t_{1} v_{1, q_{1}}^{V_{\lambda, i}} e^{i p_{1} x-\kappa_{1} x}+t_{2} v_{2, q_{2}}^{V_{\lambda, i}} e^{i p_{2} x-\kappa_{2} x}\right) e^{-i \omega_{s} t}
$$

and similarly for $\vec{A}_{\vec{x}}$ and $A_{z}$. In Eq. (28), the momenta satisfying $\omega_{1}\left(q_{1}\right)=\omega_{1}\left(q_{2}\right)=\omega_{s}$ are the complex momentum solutions for each plasmon dispersion relation with $\kappa>0$ in our geometry, fixed by the condition that modes decay infinitely far away from the interface and $\left\{t_{1}, t_{2}\right\}$ the transmission coefficients. The electromagnetic 4 -vector is converted to electric and magnetic fields through the equations

$$
\begin{aligned}
& E_{z, \lambda, i}=-\Delta_{z} V_{\lambda, i}-\partial_{t} A_{z, \lambda, i}, \\
& B_{z, \lambda, i}=-\partial_{x} A_{z, \lambda, i}+\Delta_{z} A_{x, \lambda, i} .
\end{aligned}
$$

Finally, substituting (29) in the boundary conditions (26) using Eq. (24b), we arrive at a system of four linearly coupled equations for the reflection and transmission coefficients $\left\{r_{0}, r_{\frac{2 \pi}{D}}, t_{1}, t_{2}\right\}$.

Using this approach, we recover the equilibrium JP edges [3] as shown in Fig. 8. The intuition behind this result comes from the fact that at frequencies below the lower plasmon branch there are no propagating modes available to transmit energy into the material and, as a result, the material has reflection coefficient close to unity. As soon as a propagating mode becomes available, reflectivity drops abruptly, leading to a plasmon edge.

Quasiparticle excitations both above and below $T_{c}$ will lead to dissipation of collective excitations such as JPs. We take into account effects of dissipation phenomenologically by supplementing the dispersion relation with a finite negative imaginary component:

$$
\omega(\vec{k})_{i}^{\mathrm{diss}}=\omega_{i}^{0}(\vec{k})-i \gamma_{i}(\omega),
$$

where $\omega^{\text {diss }}$ is the dispersion including dissipation, $\omega^{0}$ the dissipation-free dispersion coming from diagonalizing the Hamiltonian, and $\gamma_{i}>0$ is the mode-dependent dissipation. Such a contribution can arise physically by including a normal current component that obeys Ohm's law. The Fresnel problem is modified to finding solutions with $\omega_{i}^{\text {diss }}(\vec{k})=\omega_{s}$.

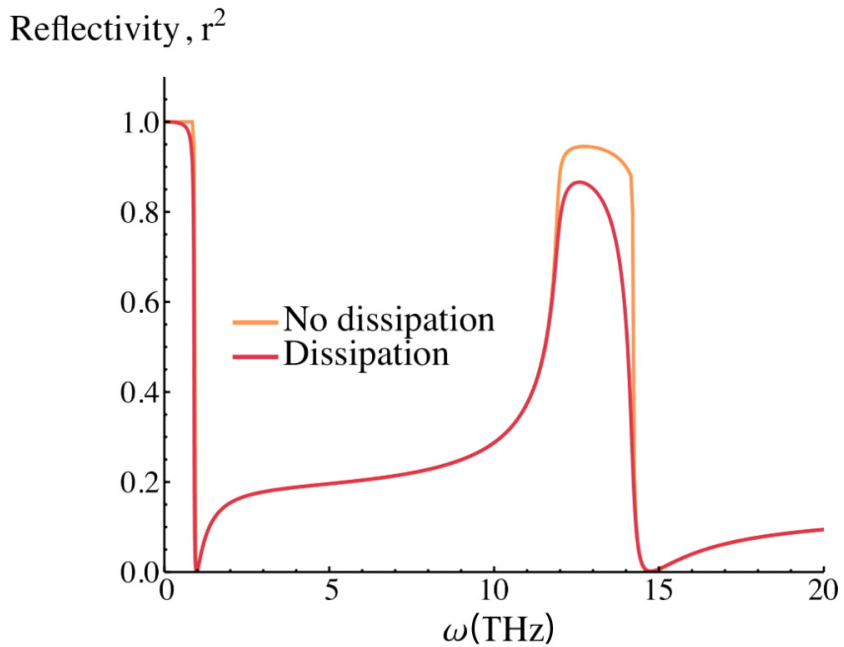

FIG. 8. Reflectivity of bilayer $\mathrm{YBa}_{2} \mathrm{Cu}_{3} \mathrm{O}_{6+x}$ as a function of frequency, with and without dissipation. At frequencies above which energy transport is permitted due to the existence of a bulk collective excitation able to transmit energy inside the material, reflectivity drops abruptly from unity. Dissipation has the effect of smoothing out the features of the dissipationless state. The two edges correspond to the two JPs.

As a result, wave vector $\vec{k}$ can be complex even when there is a bulk propagating mode due to dissipation. Reflectivity including dissipation is shown in Fig. 8, where dissipation is seen to smooth out features of the dissipationless state.

\section{PHONON-PLASMON INTERACTION}

Following the pump pulse, $\mathrm{YBa}_{2} \mathrm{Cu}_{3} \mathrm{O}_{6+\mathrm{x}}$ should develop a large coherent amplitude of the phonon operator at momentum $q=0$. In the presence of phonon-plasmon interactions excited phonons can provide parametric driving of JPs. Reflection symmetry discussed in Sec. II B plays an important role in the analysis of phonon-plasmon coupling.

Apical oxygen phonon is expected to modify the in-plane superfluid density and stiffness either by changing the inplane density of carriers or by modifying their hopping. Symmetry of the phonon mode requires that these changes are antisymmetric with respect to the two layers inside one unit cell, so that $\delta \rho_{\{1,2\}}^{\text {phon }}= \pm \tilde{\xi} Q_{\mathrm{IR}}(t) \rho_{s, 0}$ and $\delta \Lambda_{s,\{1,2\}}^{\text {phon }}(t)=$ $\pm \xi Q_{\mathrm{IR}}(t) \Lambda_{s}$, where coefficients $\tilde{\xi}$ and $\xi$ characterize the coupling strength.

Changes in the superfluid density $\delta \rho_{\{1,2\}}^{\text {phon }}$ modify Josephson coupling between the layers since $j_{c}$ should be proportional to $\sqrt{\rho_{1} \rho_{2}}$ [57]. Thus, we have $\delta j_{c, \lambda}(t)=$ $\left(\left(\tilde{\xi} Q_{\mathrm{IR}}\right)^{2}+\tilde{\xi} Q_{\mathrm{IR}}\left(\delta \rho_{2}-\delta \rho_{1}\right)\right) \frac{j_{c, \lambda}}{2 \rho_{s, 0}}$ and coupling of phonons to the out-of-plane superflow kinetic energy is given by

$$
\begin{aligned}
\delta H_{\text {kin }, z}= & \widetilde{\xi} \sum_{i} \int d^{2} x\left\{\frac{1}{2 j_{c \lambda} d_{\lambda}^{2} \rho_{s, 0}} Q(t)^{2} j_{i, \lambda, z}^{2}\right. \\
& \left.+\frac{1}{2 j_{c \lambda} d_{\lambda}^{2} \rho_{s, 0}} Q(t)\left(\delta \rho_{2}-\delta \rho_{1}\right) j_{i, \lambda, z}^{2}\right\} .
\end{aligned}
$$


Phonon-plasmon interaction in Eq. (31) describes a four-wave mixing process. The absence of a lower three-wave coupling in this case is a consequence of the fact that the Josephson couplings between the layers $j_{c, \lambda}$ are both even under reflection symmetry (19). Thus, the lowest-order coupling to odd phonons has to be quartic in the fields.

Phonon-induced changes of the superfluid stiffness modify in-plane superflow kinetic energy and give rise to additional phonon-plasmon interaction

$$
\delta H_{\mathrm{kin}, x}=\xi \sum_{i} \int d^{2} x\left\{\frac{Q_{\mathrm{IR}}(t)}{2 \Lambda_{s}}\left(j_{1, i, \vec{x}}^{2}-j_{2, i, \vec{x}}^{2}\right)\right\} .
$$

The last equation describes three-wave mixing interaction. Such a coupling is possible in this case since the superfluid stiffness of each layer $\Lambda_{s, \lambda}$ is neither odd nor even under reflection. Interaction described by Eq. (32) causes a zero-momentum three-wave parametric process that excites pairs of plasmons at opposite momenta, a process shown schematically in Fig. 1. Resonant processes that satisfy energy-matching condition $\omega_{\mathrm{ph}}=\omega_{1}(q)+\omega_{2}(-q)$ lead to exponential instability discussed in Sec. IV.

It is natural to expect that lower-order three-wave processes play a greater role in phonon-plasmon interaction. We begin the next section by providing in-depth analysis of this type of nonlinearity. We comment on the effects of quartic interactions in Eq. (31) at the end of that section.

\section{INSTABILITY ANALYSIS}

In this section we analyze the dynamical stage of the pump and probe experiments in which coherently excited phonons give rise to parametric instability of plasmons. Short-time dynamics can be deduced by treating the phonon mode as an external classical field, ignoring back action coming from plasmons. From such an analysis, we seek to find the fastest growing mode which is expected to dominate the dynamics. We project dynamics to the low-energy sector corresponding to JP relevant to parametric resonant processes involving the $\mathrm{AO}$ phonon, by expanding the original variables in the eigenbasis of the equilibrium Hamiltonian (8), using the prescription in Eq. (15) and keeping only the JPs' creation and annihilation operators. The phonon-driven plasmon Hamiltonian can then be written as

$$
\begin{aligned}
H= & \sum_{q} \sum_{i=1,2} \omega_{i}(q) b_{i}^{\dagger}(q) b_{i}(q) \\
& +\sum_{q} \sum_{i, j=1,2} g_{i, j}(t, q) b_{i}^{\dagger}(q) b_{j}^{\dagger}(-q)+\text { H.c. },
\end{aligned}
$$

where the index $i$ corresponds to the two plasmon branches and not a unit-cell index. In Eq. (33), $\omega_{i}(q)$ is the dispersion relation of the two plasmons, while the time-dependent squeezing term $g_{i, j}(q, t) b_{i}^{\dagger}(q) b_{j}^{\dagger}(q)$ arises from the parametric drive in Eq. (32) and is responsible for the exponential instability on parametric resonance. The amplitudes $g_{i, j}(q, t)=$ $\xi Q_{0} \sin \left(\omega_{\mathrm{ph}} t\right) g_{i, j}(k)$ are found from the eigenvector expansion. We simplify the discussion by considering only interband coupling since intraband processes are far detuned from resonance. This amounts to setting $g_{1,1}=g_{2,2}=0$ and $g_{1,2}(q)=g$ where for brevity the momentum dependence has been suppressed but it is always implicit. The time-dependent Hamiltonian in Eq. (33) can be turned into a time-independent infinite-dimensional banded matrix by transforming to the Floquet basis:

$$
b_{i}(t)=\int_{0}^{\omega_{\mathrm{ph}}} d \omega \sum_{n} e^{-i n \omega_{\mathrm{ph}} t-i \omega t} b_{i, n}(\omega),
$$

where $n$ is the Floquet band index. In this basis the drive appears as coupling between different Floquet bands, and the equations of motion take the form

$$
\left.\begin{array}{c}
i g^{*} \xi Q_{0} \\
-i \omega_{2}-n \omega_{\mathrm{ph}}
\end{array}\right)\left(\begin{array}{c}
b_{1, n+1}^{\dagger}(\omega) \\
b_{2, n}(\omega)
\end{array}\right)
$$

The above $2 \times 2$ matrix equation represents an infinitedimensional matrix that couples all Floquet sectors to each other. To find Floquet eigenstates, one needs to diagonalize Eq. (35). Instabilities correspond to eigenvalues with imaginary frequency components signaling exponential growth. To find the growth rate of instabilities to leading order in the drive's strength it is enough to diagonalize only two nearestneighbor Floquet bands, an approximation called Floquet degenerate perturbation theory and is equivalent to a firstorder Magnus expansion [51]. Here we sketch arguments for the validity of this approximation. Condition for parametric resonance is given by

$$
\omega_{\mathrm{ph}}=\omega_{1}+\omega_{2} .
$$

This condition makes the two different states at neighboring Floquet bands degenerate. As a result, degenerate perturbation theory can be used so that the leading contribution to the eigenvalues is given by diagonalizing the matrix only in the degenerate subspace. Note that for each $n$ there is an equivalent, decoupled, degenerate subspace and hence we can set without loss of generality $n=0$. On parametric resonance the eigenvalues of the above problem are found to be

$$
\omega=-\omega_{\{1,2\}} \pm i\left|g \xi Q_{0}\right| .
$$

Perturbative corrections in powers of $Q_{0}$ can be found by including more Floquet bands. From Eq. (37), we deduce that the growth rate is given directly by the matrix element $g \xi Q_{0}$. Away from resonance, parametrizing the detuning by $\omega_{\mathrm{ph}}=\omega_{1}+\omega_{2}+\delta$, the growth rate is given by

$$
\omega=-\left(\omega_{\{1,2\}}(q)+\frac{\delta}{2}\right) \pm i \sqrt{\left|g(q) \xi Q_{0}\right|^{2}-\left(\frac{\delta}{2}\right)^{2}},
$$

where the momentum dependence has been restored for concreteness. The detuning $\delta$ suppresses the growth rate. The leading instability is given by a plasmon pair at the wave 


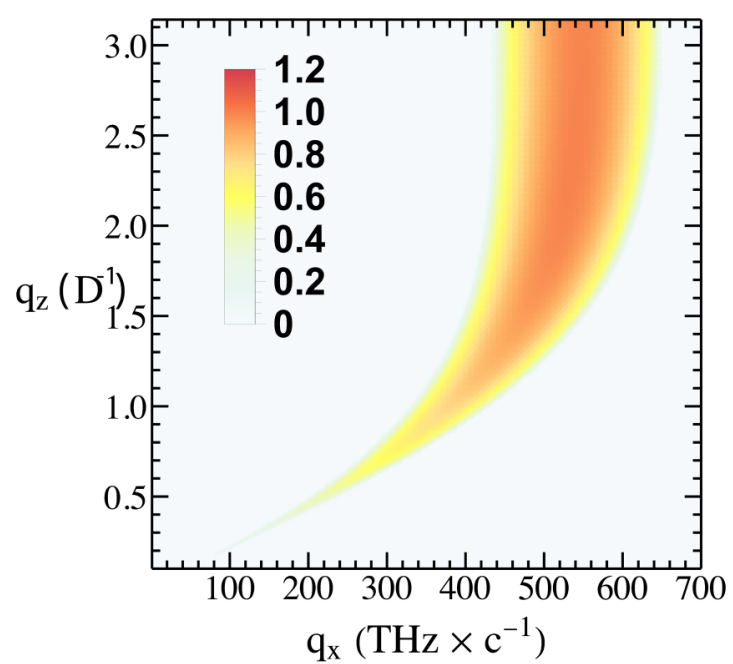

FIG. 9. Relative growth rate of unstable modes in $\left\{q_{x}, q_{z}\right\}$ plane. The relative growth rate is measured in $\mathrm{THz}$ while to obtain the true growth rate this result needs to be multiplied by the relative change in the superfluid density caused by the phonon amplitude. The coupling of phonons to the in-plane kinetic term grows quadratically with the in-plane momentum, an observation reflected by larger growth rate for instabilities with larger in-plane momentum. The most unstable JP pair is made up of a lower plasmon at $2.2 \mathrm{THz}$ and an upper plasmon at $14.3 \mathrm{THz}$.

vector which maximizes Eq. (38). Such a mode is expected to grow exponentially faster than every other mode and therefore dominate the dynamics. The relative growth rate as a function of momentum is plotted in Fig. 9. The central trace corresponds to the instability condition curve $\omega_{\mathrm{ph}}=\omega_{1}(q)+$ $\omega_{2}(q)$. As argued in Sec. III, the dominant parametric driving term comes from Eq. (32). This implies that the coupling $g(q)$ is expected to grow as $q_{x}^{2}$ while depending only weakly on $q_{z}$. Our result confirms this intuition, with the unstable mode with largest $q_{x}$ growing the fastest. Surprisingly, this occurs at the edge of the Brillouin zone along the $c$ axis at $q_{z}=\frac{\pi}{D}$. The fact that the most unstable mode occurs at the largest possible $q_{z}$ is expected to be a prediction robust to details.

Before concluding this section, we discuss our justification for not including quartic phonon-plasmon coupling described by Eq. (31). The first term in Eq. (31) can be treated in the same way as the three-wave mixing process. Since it is quadratic in the plasmon fields it can still be thought of as parametric driving of plasmons by an external field, which in this case is given by $Q^{2}(t) \rightarrow\langle Q(t)\rangle^{2}$. This process has a different resonant condition, and when the phonon amplitude $\langle Q(t)\rangle$ is small, we expect it to be subdominant. In the next section we point out, however, that this mechanism may play an important role in the renormalization of terahertz reflectivity. Another possibility is a quartic term that has one phonon and three plasmon fields. It can be studied by a different type of instability analysis (see Appendix C). Even though such a term can still lead to an instability, upon Fourier transforming this term in the momentum basis, which is the energy eigenbasis, one notes that the amplitude of the interaction is suppressed by an overall $\frac{1}{\sqrt{V}}$, where $V$ is the system's size, making this interaction irrelevant.

\section{REFLECTIVITY OF FLOQUET MEDIUM}

In the previous section we discussed a process in which a strong coherent excitation of AO phonons leads to parametric instability of JPs. Such an instability populates classically the most unstable JP modes generating supercurrents oscillating at the frequencies of these modes. Such currents have been observed by von Hoegen et al. [1] using the optical second harmonic generation technique. In this section we analyze terahertz reflectivity of the nonequilibrium state of $\mathrm{YBa}_{2} \mathrm{Cu}_{3} \mathrm{O}_{6+\mathrm{x}}$ with strongly excited phonons and Josephson plasmons. We will demonstrate that resonant parametric mode coupling naturally explains a variety of experimental signatures observed by $\mathrm{Hu}$ et al. [2] and Kaiser et al. [3]. As mentioned in the Introduction, nontrivial signatures of the phonon pump have been found both below and above $T_{c}$. Below $T_{c}$, the appearance of a photoinduced peak close to the lower JP edge and redshifting of the upper JP edge. Above $T_{c}$, the reemergence of the lower JP edge at a blueshifted frequency and the reemergence of the upper JP edge at a redshifted frequency from a featureless equilibrium spectrum in the absence of a pump.

To simplify theoretical analysis, we treat the oscillating fields as monochromatic drives that parametrically modify the equilibrium JP modes through phonon-plasmon or plasmonplasmon nonlinearities. This assumption is well justified on timescales shorter than the decay time of the oscillating modes. At longer times decay processes can be included by considering drive fields to have a range of frequencies. We postpone the discussion of the effects of the driving field's depletion until future publications. We consider the following effective model for the driven Josephson plasmons

$H_{\mathrm{eff}, \mathrm{int}}=\sum_{\alpha} \sum_{q} \sum_{i, j=1,2} g_{i, j ; \alpha} e^{i \Omega_{\mathrm{f}, \alpha} t} b_{i}^{\dagger}(q) b_{j}^{\dagger}\left(-q+Q_{\alpha}\right)+$ H.c.,

where $\alpha$ is the index of a particular oscillating field, while $\Omega_{\mathrm{fl}, \alpha}$ and $Q_{\alpha}$ are the frequency and momentum of the oscillating fields, respectively. Another underlying assumption of (39) is retaining only the pair creation processes and neglecting rescattering terms of form $e^{i \Omega_{\mathrm{f}, \alpha} t} b_{i}^{\dagger}(q) b_{j}\left(q-Q_{\alpha}\right)$. The latter are not expected to play an important role in the mechanism of parametric driving.

When analyzing terahertz reflectivity of the nonequilibrium system described by Eq. (39) we assume that for every frequency window of interest it is sufficient to include only one most relevant driving field. This corresponds to considering only the process whose frequency is closest to the parametric resonance condition. Effects coming from resonant processes are expected to strongly dominate over the nonresonant ones [58,59]. Finally, we note that symmetries impose further constraints on the allowed nonlinear processes. Here, we discuss reflection at normal angle of incidence and therefore only need to consider modes at $q_{z}=0$. An important manifestation of symmetry constraint is that the dominant three-wave mixing process that gives rise to the parametric instability of JPs (see discussion in the previous section) does not contribute to reflectivity renormalization directly: this coupling vanishes for $q_{z}=0$ because all three modes are odd 


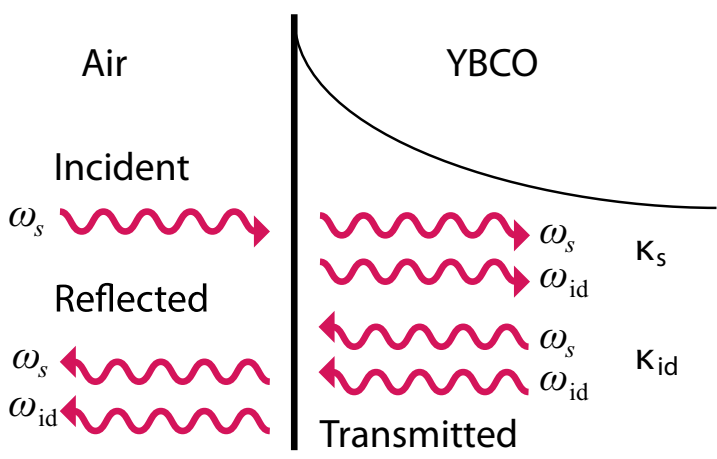

FIG. 10. Schematic reflectivity from a Floquet medium. Oscillating fields inside the medium induce frequency mixing between incident wave frequency $\omega_{s}$ and idler $\omega_{\text {idler }}$. In a Floquet medium, transmission solutions appear that propagate toward the surface amplifying reflectivity.

under the reflection symmetry discussed in Sec. II C. Another nontrivial consequence of the symmetry is that dominant nonlinear processes affecting modes close to the lower JP edge will be different compared to the one renormalizing the upper JP edge (see discussion below).

When discussing light interacting with a Floquet medium it is common to define the signal and idler frequency components where $\omega_{s}$ is the frequency of the probe and $\omega_{\text {idler }}=$ $\Omega_{\mathrm{fl}}-\omega_{s}$ ( shown schematically in Fig. 10). Within Floquet perturbation theory it is sufficient to consider mixing between these two modes and neglect the admixture of higher Floquet harmonics. To compute reflectivity, we extended the theoretical framework for solving the Fresnel problem introduced in Sec. II D for the static case to the case of a Floquet medium. Details are presented in Appendix E.

We identify three features that are expected to be ubiquitous in the reflectivity spectra of all Floquet systems and find that each experimental feature mentioned above originates from a combination of these three effects. These effects are as follows:

(i) Parametric amplification on resonance. Probing a Floquet medium at frequencies close to parametric resonance (i.e., at half the frequency of the driving field) leads to an enhancement of reflectivity and appears as a photoinduced peak.

(ii) Modes close to parametric resonance become more coherent. The exponential growth caused by the drive on parametric resonance compensates for the decay due to dissipation.

(iii) Away from parametric resonance modes are renormalized corresponding to the Floquet level attraction. As shown in Appendix D, away from resonance, states coupled through the drive are dressed by the drive. The dressed energies are shifted compared to equilibrium according to a simple level attraction rule.

We now proceed to explain each of the experimental features mentioned above.

a. Photoinduced peak in reflectivity at 1.8-2.4 THz below $T_{c}$. At normal incidence, the transmitted wave is at $q_{z}=0$. As mentioned in Sec. II, both lower and upper plasmons at $q_{z}=0$ are odd under parity. This implies that the dominant

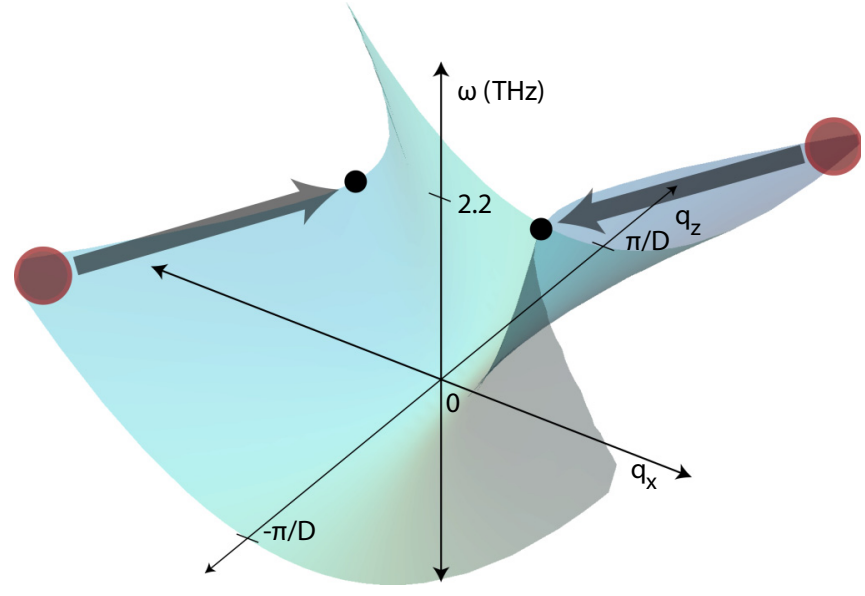

FIG. 11. Schematic representation of the possible JP four-wave mixing resonant process. A pair of parametrically unstable JPs at $q_{z}=\frac{\pi}{D}$ and $\omega=2.2 \mathrm{THz}$ can decay into a pair of JPs at $q_{z}=0$, also at $\omega=2.2 \mathrm{THz}$.

three-wave mixing process that gave rise to the parametric instability of JPs can not contribute to the reflectivity at normal incidence. On the other hand, the four-wave mixing process ignored in the last section can now contribute and corresponds to a parametric drive with amplitude $\left\langle Q_{1}(t)\right\rangle\left\langle Q_{2}(t)\right\rangle$, shown schematically in Fig. 2(a). Such a drive oscillates at a frequency corresponding to the difference between phonon $Q_{1}$ at $\sim 16.5 \mathrm{THz}$ and the adjacent phonon $Q_{2}$ at $\sim 20.4 \mathrm{THz}$ which is also excited during the pump process as reported in experiments of Hu et al. [2] and Kaiser et al. [3]. This leads to a drive at zero momentum and frequency $\omega_{d}=3.9 \mathrm{THz}$. Another possible mechanism is a four-wave mixing process between JPs shown in Fig. 11. JPs at frequency $2.2 \mathrm{THz}$ and $q_{z}=\frac{\pi}{D}$, excited by $\mathrm{AO}$ phonons through the parametric resonance process mentioned in the previous section, can themselves become a source of parametric driving. For example, they can drive the lower JPs at $q_{z}=0$, which play the key role in light reflection near the lower JP edge. A similar mechanism has been observed by Baumberg et al. for excitons near the so-called magic angle [60]. Both mechanisms provide a parametric drive at $q=0$ but at slightly different frequencies (3.9 THz for the phonon mechanism and $4.4 \mathrm{THz}$ for the JP mechanism). As we will discuss below, both scenarios produce similar results and are consistent with experimental data. We focus here on the phonon four-wave mixing mechanism. In this scenario, the effective interacting Hamiltonian keeping only resonant time-dependent contributions is given by

$$
H_{\text {eff, int }}=\sum_{q} g_{q}^{\text {lower }}\left\langle Q_{1}(t)\right\rangle\left\langle Q_{2}(t)\right\rangle b_{1}^{\dagger}(q) b_{1}^{\dagger}(-q) .
$$

In the absence of dissipation, reflectivity as a function of frequency in the presence of this phonon four-wave mixing term is plotted in Fig. 12. The most striking feature is the appearance of a photoinduced peak at the parametrically resonant frequency $\frac{\omega_{d}}{2}=2 \mathrm{THz}$. This suggests that the experimental feature of a photoinduced peak arises from parametric amplification of reflectivity due to the four-wave phonon-plasmon process. 


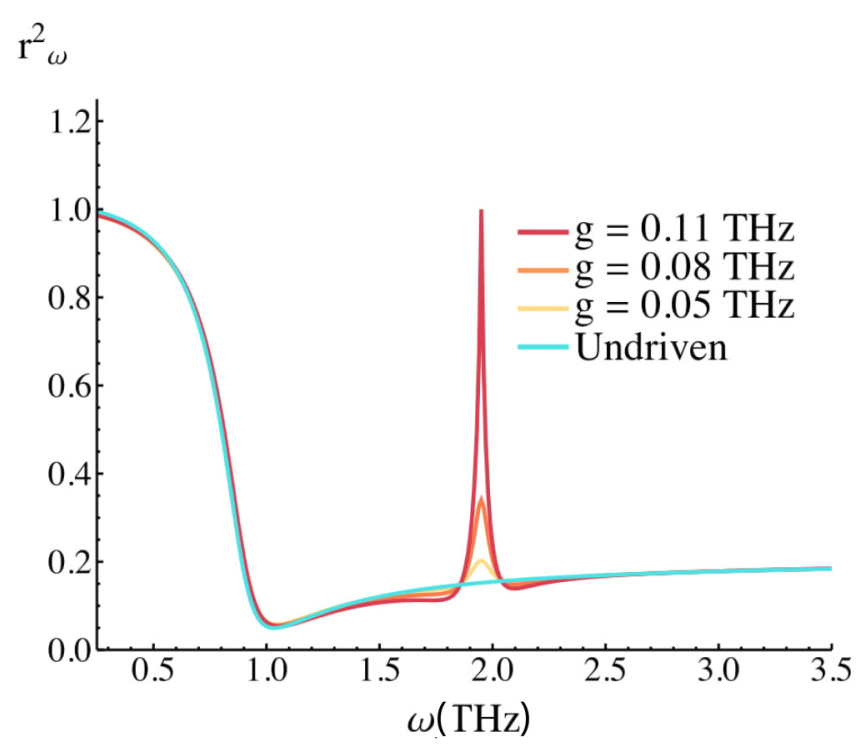

FIG. 12. Reflectivity at the probe frequency as a function of frequency for Floquet $\mathrm{YBa}_{2} \mathrm{Cu}_{3} \mathrm{O}_{6+\mathrm{x}}$ after the pump in the regime of small dissipation. The leading oscillating term considered is a two-phonon four-wave mixing process oscillating at the difference of two adjacent phonon modes at $\Omega_{\mathrm{fl}}=3.9 \mathrm{THz}$. As the amplitude of oscillation is increased, a photoinduced peak appears on parametric resonance, $\omega=2 \mathrm{THz}$. Another possible mechanism of this peak is four-plasmon resonant scattering shown in Fig. 11.

b. Reemergence of lower JP edge at 1.8-2.4 THz above $T_{c}$. We now address the question of the emergence of the lower JP edge in the pseudogap regime reported by $\mathrm{Hu}$ et al. [2] and Kaiser et al. [3]. We assume that at equilibrium in this regime, JPs can be described as overdamped modes which do not produce a plasmon edge. The situation for strong dissipation $\gamma_{1}=1 \mathrm{THz}$ [see Eq. (30)] is shown in Fig. 13. In the absence of a drive, reflectivity is featureless. However,

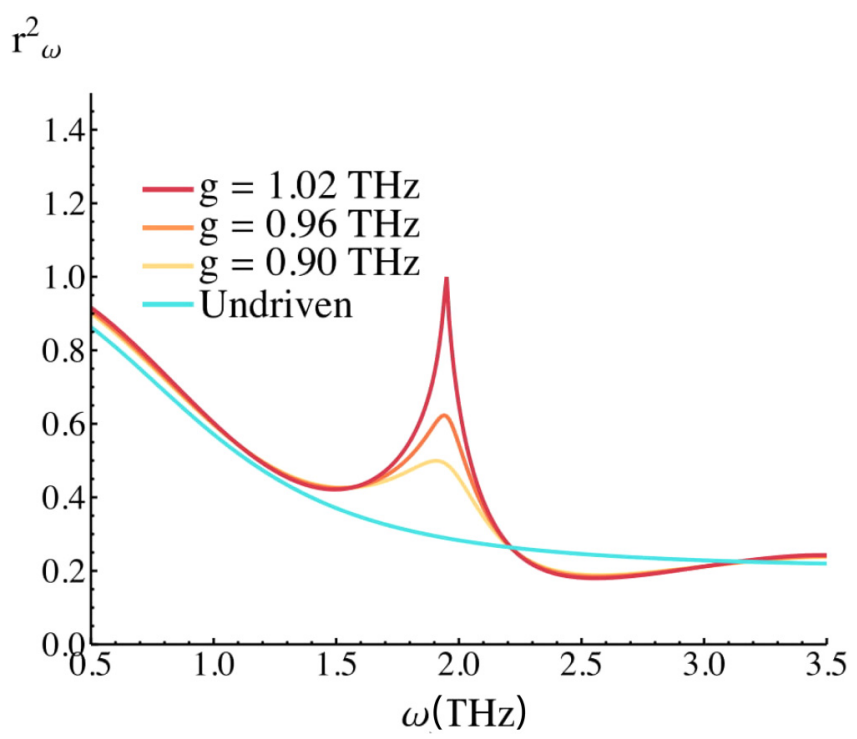

FIG. 13. Reflectivity of Floquet medium in the overdamped case. Parametric amplification of the probe beam at $2 \mathrm{THz}$ reestablishes the lower plasmon edge at a blueshifted frequency $\omega=2 \mathrm{THz}$.

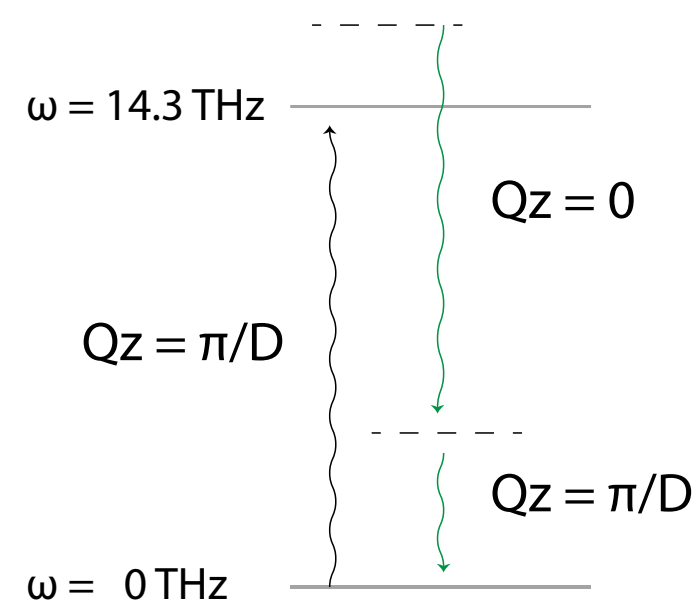

FIG. 14. Schematic representation of the three-wave mixing process renormalizing the upper plasmon edge. The instability-induced pump corresponding to the expectation value of the upper plasmon at $Q_{z}=\frac{\pi}{D}, Q_{x}=500 \mathrm{THz} / \mathrm{c}$, and $\omega=14.3 \mathrm{THz}$ acts as an external drive (black). This drive couples the upper plasmon band at $Q_{z}=0$ (green) to the lower plasmon band at $Q_{z}=\frac{\pi}{D}$ (green). However, the sum of the energies of the upper and lower plasmons is higher than $\omega=14.3 \mathrm{THz}$ which leads to a redshift of the upper plasmon.

for strong enough drive the plasmon edge reemerges at the blueshifted frequency around $\frac{\omega_{d}}{2}$ where parametric driving compensates dissipation most efficiently reviving features of the dissipationless state.

In the case of the JP four-wave mixing mechanism shown in Fig. 11, the results would be the same but with a slightly shifted parametric resonance frequency at $\frac{\omega_{d}}{2}=2.2 \mathrm{THz}$. The experimental results place the above features at $1.8-2.4 \mathrm{THz}$, thus both scenarios are consistent with these experiments. One way to determine which mechanism dominates is to pump the system with a narrow-bandwidth pulse. If only one of the phonons is excited but the feature remains, this would be evidence that the JP four-wave mixing process is responsible for this reflectivity feature instead of the phonon four-wave mixing process.

c. Redshift of upper JP edge below $T_{c}$. At frequencies around $14 \mathrm{THz}$ corresponding to the upper plasmon edge, the four-wave mixing term involving two phonons mentioned in the previous section is far detuned. In this frequency window, the resonant process that obeys the parity symmetry constraints is a three-wave mixing process between JPs. This process involves the upper plasmon at $Q_{z}=\frac{\pi}{D}$ and $Q_{x}=$ $500 \mathrm{THz} / \mathrm{c}$ and oscillating at $14.3 \mathrm{THz}$ converted into an upper plasmon at $q_{z}=0$ and a lower plasmon at $q_{z}=\frac{\pi}{D}$ to conserve momentum, shown schematically in Fig. 14. The upper plasmon at $Q_{z}=\frac{\pi}{D}$ and $Q_{x}=500 \mathrm{THz} / \mathrm{c}$ corresponds to an unstable mode which is classically populated and is treated as an external drive. In this scenario, the effective interacting Hamiltonian takes the form

$$
\begin{aligned}
H_{\text {eff,int }}= & \sum_{q_{x}} g_{q}^{\text {upper }}\left\langle b_{2}\left(Q_{z}, Q_{x}\right)\right\rangle \\
& \times b_{2}^{\dagger}\left(q_{z}=0, q_{x}\right) b_{1}^{\dagger}\left(Q_{z}, Q_{x}-q_{x}\right) .
\end{aligned}
$$

In Eq. (41), we consider the upper plasmon mode $b_{2}\left(q_{z}=\right.$ $\left.0, q_{x}\right)$, which couples to the incoming probe beam at nor- 


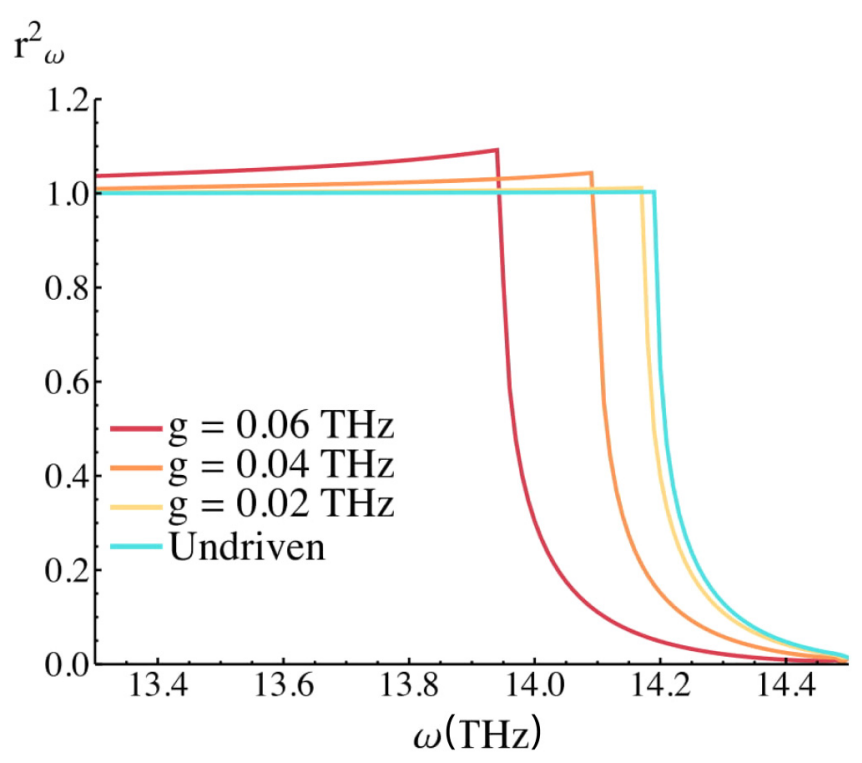

FIG. 15. Reflectivity excluding the idler component as a function of frequency of the Floquet medium with weak dissipation, with $\Omega_{\mathrm{fl}}=14.3 \mathrm{THz}$ at different driving amplitudes. The upper plasmon edge is redshifted.

mal incidence whose momentum along the $c$ axis is fixed to $q_{z}=0$ from boundary conditions. The upper plasmon at $Q=\left\{Q_{z}, Q_{x}\right\}$ has a classical expectation value and acts as an external drive. Finally, to preserve both momentum conservation and parity conservation, the mode coupled to the probe beam is dressed through the drive with the state $b_{1}^{\dagger}\left(Q_{z}, Q_{x}-\right.$ $q_{x}$ ) renormalizing its energy. Level attraction (Appendix D) implies that this parametric driving process will result in a redshift of the upper plasmon edge as the sum of the bottom of the two bands $(0.9 \mathrm{THz}+14.2 \mathrm{THz})$ is greater than the frequency of the drive $(14.3 \mathrm{THz})$. This situation is demonstrated in Fig. 15.

d. Reemergence of upper JP edge at redshifted frequency above $T_{c}$. The pseudogap phase is modeled phenomenologically through strong dissipation as in the previous discussion. The reflectivity in the pumped state with strong dissipation is plotted in Fig. 16 and shows clear indication of a reemergence of the upper JP edge at a redshifted frequency in agreement with the experiments. This implies that this experimental signature is the result of parametric driving making equilibrium modes more coherent close to resonance and the energy shift caused by level attraction.

\section{CONCLUSIONS AND OUTLOOK}

In summary, we developed a unified theoretical model that describes pump and probe experiments in $\mathrm{YBa}_{2} \mathrm{Cu}_{3} \mathrm{O}_{6+\mathrm{x}}$, in which resonant excitation of apical oxygen phonons leads to dramatic changes in the low-frequency reflectivity both below the superconducting $T_{c}$ and in the pseudogap phase. Our analysis provides a general framework for understanding generation of currents following a pump resonant to the AO phonons, as well as reemergence of the lower plasmon edge above $T_{c}$ at blueshifted frequency and of the upper plasmon edge at a redshifted frequency. Finally, we were able to ex-

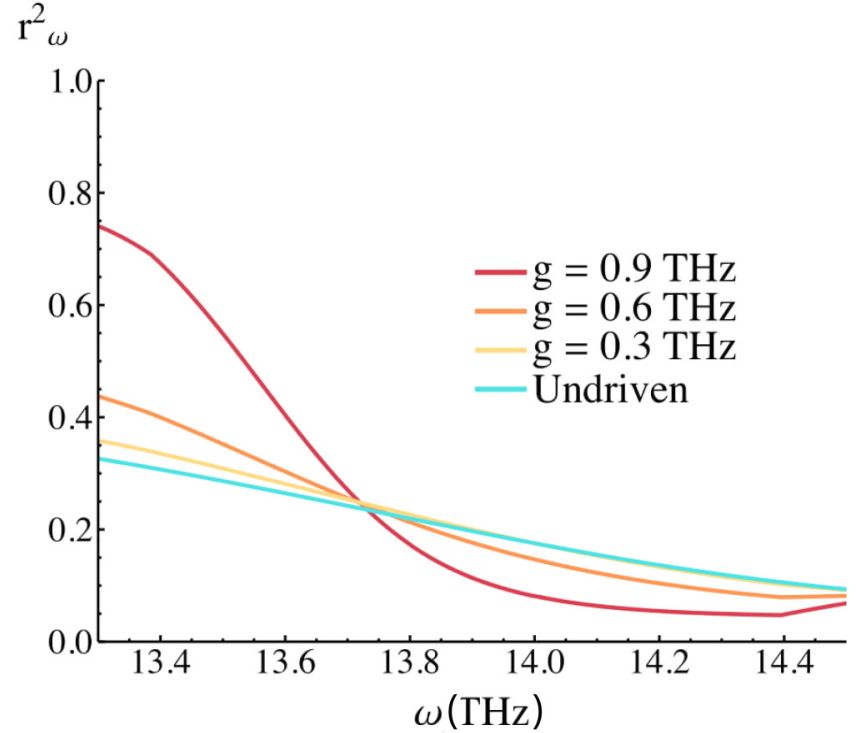

FIG. 16. Reflectivity at probe frequency near the upper plasmon edge as a function of frequency in the presence of dissipation. Parametric driving from the upper-plasmon-induced pump revives the upper plasmon edge at a redshifted value.

plain the appearance of a photoinduced peak in the reflectivity below $T_{c}$ and the renormalization of the upper plasmon edge to lower frequencies. We demonstrated that the origin of all these phenomena is in the nonlinear couplings between phonons and Josephson plasmons, with the first step being parametric excitation of pairs of Josephson plasmons by strongly photoexcited AO phonons. The latter process originates from a renormalization of the in-plane superfluid density due to the apical oxygen's motion; for such a coupling the parametric resonance condition can be satisfied for pairs of plasmons in the upper and lower branches at opposite momenta. This analysis allowed us to compute the growth rate of JP pairs in the full $q_{\|}, q_{z}$ plane. Our results identify an instability curve in the momentum plane for the unstable plasmons which lies outside the light cone. Subsequently, we proceeded to calculate reflectivity in equilibrium and in the pumped state. In equilibrium, we developed a framework to solve the Fresnel problem in a bilayer systems such as $\mathrm{YBa}_{2} \mathrm{Cu}_{3} \mathrm{O}_{6+\mathrm{x}}$. This framework was extended to a Fresnel-Floquet problem which takes into account the effects of an oscillating field using Floquet degenerate perturbation theory.

We suggested that the pseudogap phase can be understood as hosting overdamped plasmons, and discussed that this scenario provides a good description for the observed phenomena of photoinduced superconductivity in $\mathrm{YBa}_{2} \mathrm{Cu}_{3} \mathrm{O}_{6+\mathrm{x}}$. Going beyond phenomenological description of this regime is beyond the scope of this paper. Such analysis can be done by extending earlier work on Josephson plasmons in the vortex liquid phase $[35,39,41,42,61,62]$. Thermal fluctuations of the pairing amplitude are expected to lead to plasmon broadening and suppression of the parametric instability [35]. Hence, detailed analysis of phonon-plasmon three-wave parametric resonance above $T_{c}$ should provide valuable insight into the nature of superconducting fluctuations in the pseudogap 
regime [26,63-65]. We plan to present such an analysis in subsequent publications.

Before concluding this paper, we would like to point out that plasmons generated from photoexcited phonons through parametric instability do not radiate out of the sample because of the mismatch of energy and momentum with electromagnetic waves in vacuum. However, they can be made to radiate into the far field, if one adds a metamaterial-type structure on the surface of a sample, which compensates for the momentum mismatch (see, e.g., structures in references [66,67]). By varying the spatial periodicity of such structures, one should be able to enhance emission of Josephson plasmons at different momenta. This opens a promising new direction for generation of terahertz waves as entangled pairs with tunable frequencies.

\section{ACKNOWLEDGMENTS}

We are grateful for useful discussions with P. Dolgirev, K. Seetharam, L. Glazman, I. Klich, P. Lee, B. I. Halperin, D. Podolsky, S. Sugiura, J. Orenstein, M. Mitrano, D. Jaksch, R. Averitt, A. Millis, A. Georges, T. Giamarchi, R. Citra, M. Knap, and E. Orignac. M.H.M. and E.D. acknowledge support from Harvard-MIT CUA, AFOSR-MURI: Photonic Quantum Matter (Award No. FA95501610323), DARPA DRINQS program (Award No. D18AC00014). A.vH., M.F, M.F, and A.C. acknowledge funding from the European Research Council under the European Union's Seventh Framework Programme (FP7/2007- 2013)/ERC Grant Agreement No. 319286 (Q-MAC). A.C. acknowledges support from the Deutsche Forschungsgemeinschaft (DFG) via the Cluster of Excellence "The Hamburg Centre for Ultrafast Imaging" (EXC 1074-Project ID No. 194651731) and from the priority program SFB925.

\section{APPENDIX A: MATHEMATICAL DETAILS OF DIAGONALIZATION PROCEDURE}

In this paper we use a generalized Bogoliubov transformation in order to transform the Hamiltonian in Eq. (8) written in terms canonically conjugate pairs with generalized coordinates $\vec{q}_{i}=\left\{\rho_{i, \lambda}, V_{i, \lambda}, \vec{A}_{\vec{x}}, A_{z, i, \lambda}\right\}$ and conjugate momenta $\vec{p}_{i}=$ $\left\{\phi_{i, \lambda}, P_{V_{i, \lambda}}, P_{\vec{A}_{\vec{x}}}, P_{A_{z, i, \lambda}}\right\}$ to a diagonal Hamiltonian written in terms of creation and annihilation operators for the elementary excitations of the system (plasmons). Our notation follows closely Ref. [56]. We define the spatial Fourier transform as

$$
\begin{aligned}
& q_{i}(\vec{x})=\int_{-\frac{\pi}{D}}^{\frac{\pi}{D}} \frac{d k_{z}}{\sqrt{2 \pi}} \int \frac{d^{2} k_{x}}{2 \pi} e^{i \vec{k}_{x} \vec{x}+i k_{z} D i} q(\vec{k}), \\
& p_{i}(\vec{x})=\int_{-\frac{\pi}{D}}^{\frac{\pi}{D}} \frac{d k_{z}}{\sqrt{2 \pi}} \int \frac{d^{2} k_{x}}{2 \pi} e^{i \vec{k}_{x} \vec{x}+i k_{z} D i} p(\vec{k}) .
\end{aligned}
$$

The Hamiltonian is translationally invariant and therefore diagonal in momentum space and takes the form

$$
H=\int_{-\frac{\pi}{D}}^{\frac{\pi}{D}} \frac{d k_{z}}{\sqrt{2 \pi}} \int \frac{d^{2} k_{x}}{2 \pi}\left(\frac{1}{2} \phi^{\dagger}(\vec{k}) \cdot A(k) \cdot \phi(\vec{k})\right),
$$

where $A$ is a Hermitian matrix. In Eq. (A2), the vectors $\phi^{\dagger}(\vec{k})$ and $\phi(\vec{k})$ are defined as

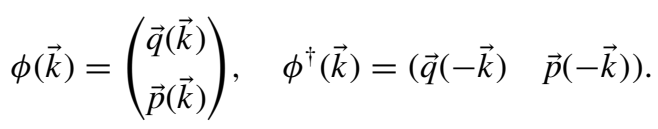

The equations of motion of $\phi(\vec{k})$ are given through the commutation relations, written here in compact matrix notation:

$$
\left[\phi(\vec{k}), \phi^{\dagger}\left(\vec{k}^{\prime}\right)\right]=-\Sigma_{y} \delta^{3}\left(\vec{k}-\delta k^{\prime}\right),
$$

where $\Sigma_{y}=\left(\begin{array}{cc}0 & -i I_{8} \\ i I_{8} & 0\end{array}\right)$ encodes the canonical commutation relations of $q$ and $p$ and $I_{8}$ is the eight-dimensional identity matrix. The equations of motion for $\phi(\vec{k})$ are then given by computing Heisenberg equations of motion:

$$
i \frac{d}{d t} \phi(\vec{k})=M(\vec{k}) \phi(\vec{k})
$$

where $M(\vec{k})=-\Sigma_{y} A(\vec{k})$ is the dynamical matrix defined in Eq. (11) of the main text. Looking for oscillating solutions in time $\phi(\vec{k}, t)=\phi(\vec{k}) e^{-i \omega t}$ leads to the eigenvalue equation

$$
\omega \vec{v}(\vec{k}, \omega)=M(\vec{k}) \vec{v}(\vec{k}, \omega) .
$$

It can been proven [56] that for real eigenvalues the eigenvectors of $M(\vec{q})$ form an orthonormal basis under the inner product $-\Sigma_{y}$, i.e.,

$$
\left\langle\left. v\left(\omega_{i}\right)\left|v\left(\omega_{j}\right\rangle \equiv v\left(\omega_{i}\right)^{\dagger}\left(-\Sigma_{y}\right) v\left(\omega_{j}\right)=\right| v\left(\omega_{i}\right)\right|^{2} \delta_{i, j} .\right.
$$

Moreover, if $v(\omega)$ is an eigenvector with eigenvalue $\omega$ then $v(\omega)^{*}$ is also an eigenvector with eigenvalue $-\omega$ and opposite norm:

$$
v(\omega)^{\dagger}\left(-\Sigma_{y}\right) v(\omega)=-\left(v(-\omega)^{\dagger}\left(-\Sigma_{y}\right) v(-\omega)\right)^{*} .
$$

As a result, one can order eigenvectors in such a way that positive-frequency positive-norm eigenvectors appear first and negative-frequency negative-norm eigenvectors appear second:

$$
T_{d}=\left\{v\left(\omega_{1}\right), \ldots, v\left(\omega_{n}\right), v\left(-\omega_{1}\right), \ldots, v\left(-\omega_{n}\right)\right\} .
$$

$T_{d}$ is the transformation matrix that relates canonically conjugated pairs to creation and annihilation operators:

$$
\phi(\vec{k})=T_{d}(\vec{k}) \psi(\vec{k}) .
$$

$\psi(\vec{k})$ contains the bosonic creation and annihilation operators that correspond to each eigenvector:

$$
\psi(\vec{k})=\left(\begin{array}{c}
b_{1}(\vec{k}) \\
\vdots \\
b_{1}^{\dagger}(-\vec{k}) \\
\vdots
\end{array}\right) .
$$

The orthonormality condition on the eigenvectors can be summarized using the matrix equation

$$
T_{d}^{\dagger}\left(-\Sigma_{y}\right) T_{d}=\Sigma_{z}
$$

with $\Sigma_{z}=\left(\begin{array}{cc}I_{4} & 0 \\ 0 & -I_{4}\end{array}\right)$, where we remind the reader that we include only eigenvectors that correspond to physical degrees of freedom which reduces the dimensionality of the system 


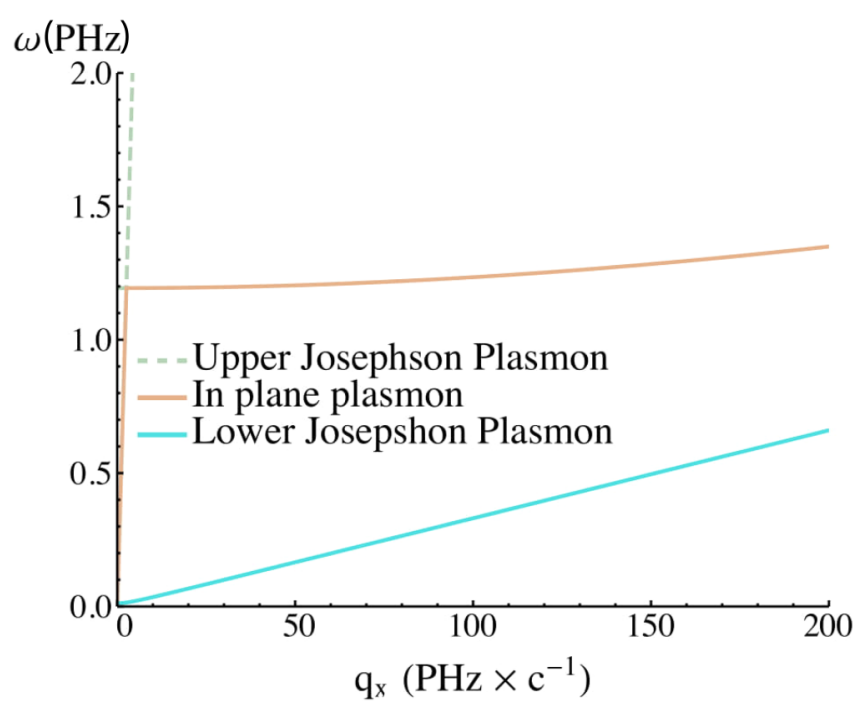

FIG. 17. Dispersion of the three plasmons in the nonrelativistic regime. The upper plasmon increases almost vertically due to the large speed of light. The remaining two plasmons increase linearly in energy as a function of $q_{x}$ for large $q_{x}$ due to the in-plane kinetic energy contribution.

from 16 to 8 . One can confirm that the above normalization conditions on the eigenvectors imply that $\psi(\vec{k})$ obeys the usual commutation relations

$$
\left[\psi(\vec{k}), \psi^{\dagger}(\vec{k})\right]=\Sigma_{z} .
$$

This mathematical structure underlies the majority of our work and allows us to project dynamics lowest-energy subspace corresponding to JPs. Given an arbitrary function involving the state vector $\phi(\vec{k})$, dynamics can be projected to the JP subspace $\left\{b_{1}, b_{2}, b_{1}^{\dagger}, b_{2}^{\dagger}\right\}$ by projecting each state vector inside the function

$$
\begin{aligned}
\mathcal{P}_{\mathrm{JP}} \cdot \phi(\vec{k}) & =c_{1} b_{1}+c_{2} b_{2}+c_{1}^{\dagger} b_{1}^{\dagger}+c_{2}^{\dagger} b_{2}^{\dagger}, \\
c_{i} & =v\left(\omega_{i}(\vec{k})\right) \cdot\left(-\Sigma_{y}\right) \cdot \phi(\vec{k}) .
\end{aligned}
$$

\section{APPENDIX B: DISPERSION OF PLASMONS IN THE NONRELATIVISTIC LIMIT}

In this Appendix, we show the dispersion of the system in the nonrelativistic limit for all three plasmons in our system, shown in Fig. 17. Most notably, adopting a nonrelativistic approach which replaces electric potentials with Coulomb's law ignores the upper JP along the $q_{x}$ axis which corresponds to a photon. Photon excitations are ignored in nonrelativistic treatments because at large momenta $q_{x} \gg \frac{1}{c} \sqrt{\Lambda_{s} \frac{\left(e^{*}\right)^{2}}{\epsilon}}$ the dispersion grows linearly with the large speed of light and these modes correspond to very high-energy excitations (seen in Fig. 17 as a linear dispersion excitation with a large gradient in green). Our formalism interpolates between the relativistic regime presented in the main paper, which participates in the parametric amplification process and the nonrelativistic regime which is often employed in solid-state physics. In the nonrelativistic limit, corrections from the in-plane kinetic energy offer significant contributions to the lower plasmon, which instead of saturating as predicted by previous work, it instead grows linearly.

\section{APPENDIX C: FOUR-WAVE MIXING PARAMETRIC RESONANCE}

In this Appendix we perform an instability analysis for four-wave mixing process where one phonon acting as the external drive is converted into three JPs. This process is represented as a cubic interaction with a time-dependent coupling. We show in this Appendix that the same intuition can be used for four-wave mixing as with three-wave mixing processes. Instead of considering only equations of motion on one-point functions, e.g., $\langle b\rangle$, as is the case for normal parametric resonance, to treat a fluctuating interacting we need to include two point functions as well, e.g., $\langle b b\rangle$, and perform a stability analysis around the equilibrium state. This amounts to performing an instability analysis around coherent state perturbations for which $\langle b\rangle \neq 0$, coupled to squeezed states for which $\langle b b\rangle \neq 0$. Without loss of generality, we consider a three-mode Hamiltonian with nonlinear driving that couples all three of them:

$$
H=\sum_{i} \omega_{i} b_{i}^{\dagger} b_{i}+2 \epsilon \cos (\Omega t)\left(b_{1}^{\dagger} b_{2}^{\dagger} b_{3}^{\dagger}+\text { H.c. }\right) .
$$

Notice that a particular form of cubic driving has been used including only terms that include three creation or three annihilation operators. One can confirm that all possible other combinations will not lead to an instability of the ground state. Linearizing around the undriven ground state, the equations of motion take the form

$$
\begin{aligned}
\frac{d\left\langle b_{1}^{\dagger}\right\rangle}{d t} & =i \omega_{1}\left\langle b_{1}^{\dagger}\right\rangle+i \epsilon 2 \cos (\Omega t)\left\langle b_{2} b_{3}\right\rangle, \\
\frac{d\left\langle b_{2} b_{3}\right\rangle}{d t} & =-i\left(\omega_{2}+\omega_{3}\right)\left\langle b_{2} b_{3}\right\rangle-i \epsilon 2 \cos (\Omega t)\left\langle b_{1}^{\dagger}\right\rangle .
\end{aligned}
$$

These equations along with their permuted counterparts $(\{1,2,3\} \rightarrow\{3,1,2\} \rightarrow\{2,3,1\})$, will form the degenerate subspace in Floquet perturbation theory on resonance (see Sec. IV):

$$
\Omega=\omega_{1}+\omega_{2}+\omega_{3} .
$$

Using the Floquet ansatz introduced in Eq. (34), the resulting secular equation has eigenfrequency

$$
\omega=-\omega_{2} \pm i \epsilon .
$$

As for three-wave mixing process, the time-dependent coupling leads to an instability on resonance with a growth rate that corresponds to the nonlinear amplitude $\epsilon$. For extended states, the nonlinear amplitude is proportional to $\epsilon \propto \frac{1}{\sqrt{V}}$. This suppression makes it a subleading process compared to three-wave mixing terms.

Finally, we would like to stress that this process has no classical analog. The resonant pair corresponds to a coherent state coupled to a squeezed state. However, since this occurs for every mode in the resonant trio independently and at the same rate, we do not expect a true squeezed state to emerge 
unless a specific coherent state has been biased by an experimental probe.

\section{APPENDIX D: DRESSED STATE}

In the main text, we report that, away form resonance, states are shifted in energy as a result of the drive according to a simple level attraction rule. The level attraction rule is stated as follows: For two modes at frequencies $\omega_{1}(q)$ and $\omega_{2}(-q+Q)$ coupled through a drive with frequency $\Omega_{\mathrm{fl}}$ and momentum $Q$, the energies of the modes become UV shifted when the condition $\omega_{1}(q)+\omega_{2}(-q+Q)<\Omega_{\mathrm{fl}}$ is satisfied and IR shifted for states that obey $\omega_{1}(q)+\omega_{2}(-q+Q)<$ $\Omega_{\mathrm{fl}}$. This a consequence of Floquet perturbation theory which can be applied away from resonance. Under parametric driving the Hamiltonian for the two coupled modes takes the form

$$
\begin{aligned}
H_{q}= & \omega_{1, q} b_{1, q}^{\dagger} b_{1, q}+\omega_{2, q-Q} b_{2, Q-q}^{\dagger} b_{2, Q-q} \\
& +\left(\lambda e^{-i \Omega_{\mathrm{fl}} t} b_{q, 1}^{\dagger} b_{2, Q-q}^{\dagger}+\text { H.c. }\right) .
\end{aligned}
$$

This Hamiltonian is turned into a time-independent Hamiltonian through a suitable rotating frame transformation:

$$
U=\exp \left\{-i \Omega_{\mathrm{fl}} b_{1, q}^{\dagger} b_{1, q}\right\} .
$$

The effect of this transformation is given by

$$
U b_{1, q} U^{\dagger}=e^{-i \Omega_{\mathrm{fl}} t} b_{1, q}
$$

and the rotating frame Hamiltonian is found to be

$$
\begin{aligned}
H^{\prime}= & U H U^{\dagger}+i U \partial_{t} U^{\dagger} \\
= & \left(\omega_{1, q}-\Omega_{\mathrm{fl}}\right) b_{1, q}^{\dagger} b_{1, q}+\left(\omega_{2, q-Q}\right) b_{2, Q-q}^{\dagger} b_{2, Q-q} \\
& +\left(\lambda b_{q, 1}^{\dagger} b_{2, Q-q}^{\dagger}+\text { H.c. }\right) .
\end{aligned}
$$

We now compute the dressing of the vacuum and excited modes due to the parametric drive. The vacuum is dressed as

$$
|\tilde{0}\rangle=|0\rangle+\frac{g}{\Omega_{\mathrm{fl}}-\omega_{1, q}-\omega_{2, q-Q}} b_{1, q}^{\dagger} b_{2, Q-q}^{\dagger}|0\rangle .
$$

The dressed vacuum energy becomes

$$
\widetilde{E}_{0}=\frac{|g|^{2}}{\Omega_{\mathrm{fl}}-\omega_{1, q}-\omega_{2, q-Q},}
$$

while the first excited state for each mode becomes

$$
\begin{array}{r}
|\widetilde{1}\rangle_{1, q}=b_{1, q}^{\dagger}|0\rangle+\frac{\sqrt{2} g}{\Omega_{\mathrm{fl}}-\omega_{1, q}-\omega_{2, q-Q}}\left(b_{1, q}^{\dagger}\right)^{2} b_{2, Q-q}^{\dagger}|0\rangle, \\
|\widetilde{1}\rangle_{2, Q-q}=b_{2, Q-q}^{\dagger}|0\rangle+\frac{\sqrt{2} g}{\Omega_{\mathrm{fl}}-\omega_{1, q}-\omega_{2, q-Q}}\left(b_{2, Q-q}^{\dagger}\right)^{2} b_{1, q}^{\dagger}|0\rangle
\end{array}
$$

whose energy is shifted by

$$
\begin{gathered}
\widetilde{E}_{1, q}^{(1)}=\omega_{1, q}+\frac{2|g|^{2}}{\Omega_{\mathrm{fl}}-\omega_{1, q}-\omega_{2,-q}}, \\
\widetilde{E}_{2,-q}^{(1)}=\omega_{2,-q}+\frac{2|g|^{2}}{\Omega_{\mathrm{fl}}-\omega_{1, q}-\omega_{2,-q}} .
\end{gathered}
$$

As a result, the renormalized energy of a single plasmon excitation in either band is shifted by the same amount,

$$
\widetilde{\omega}_{i}=\widetilde{E}_{i}^{(1)}-\widetilde{E}^{(0)}=\omega_{i}+\frac{|g|^{2}}{\Omega_{\mathrm{fl}}-\omega_{1, q}-\omega_{2, q-Q}},
$$

where $i=\{(1, q),(2,-q)\}$. Following a similar analysis it is easy to check that this applies to all higher-energy states:

$$
\widetilde{E}_{i}^{(n+1)}-\widetilde{E}_{i}^{(n)}=\omega_{i}+\frac{|g|^{2}}{\Omega_{\mathrm{fl}}-\omega_{1, q}-\omega_{2,-q}} .
$$

From this analysis we conclude that generically due to a parametric drive, a pair of states below parametric resonance $\omega_{1, q}+\omega_{2, q-Q}<\Omega_{\mathrm{fl}}$ are UV shifted while states above parametric resonance $\omega_{1, q}+\omega_{2, q-Q}>\Omega_{\mathrm{fl}}$ are IR shifted.

\section{APPENDIX E: FLOQUET FRESNEL PROBLEM}

In this Appendix we will provide a general framework for calculating reflectivity in the presence of a periodic drive. We will keep the discussion general and allow for the periodic drive to have finite momentum. Reflectivity in a periodically driven system can be calculated by finding first the Floquet eigenstates oscillating at the frequency of the incident beam $\omega_{s}$. Describing the Floquet drive by the effective Hamiltonian in Eq. (39) is equivalent to starting from the equations of motion in the original variables with the a time-periodic perturbation

$$
i \partial_{t} \vec{v}=\left[M_{0}+M_{1} \cos \left(\Omega_{\mathrm{fl}} t\right)\right] \vec{v}
$$

and projecting the driving matrix $M_{1}$ to the subspace of the JPs, which correspond to the lowest-energy eigenstates of the equilibrium matrix $M_{0}$. Under a time-periodic drive, eigenvectors of the equations of motions have the Floquet form

$$
\vec{v}(\vec{x}, t)=\sum_{i, n} e^{i n \Omega_{\mathrm{f}} t-i \omega t+i q_{n} x} v_{i}\left(\vec{q}_{n}\right),
$$

where $v_{i}\left(q_{n}\right)$ is a shorthand notation for the equilibrium eigenvectors that obey

$$
\omega_{i}\left(\vec{q}_{n}\right) \vec{v}_{i}\left(\vec{q}_{n}\right)=M_{0} \vec{v}_{i}\left(\vec{q}_{n}\right),
$$

and $i$ labels the plasmon branch. In Eq. (E2), $\vec{q}_{n}$ reflects the fact that $M_{1}$ can in principle break translational invariance and mix states with different momenta, as is the case when the pump originates from a finite-momentum JP instability. Assuming the driving amplitude is small, the above sum can be restricted to combination of modes that are coupled resonantly only within the nearest-neighboring Floquet bands. In other words, we include only the signal $\omega_{s}$ and idler $\omega_{\text {idler }}=$ $\Omega_{\mathrm{fl}}-\omega_{s}$ frequency components in the ansatz to diagonalize Eq. (E1):

$$
\vec{v}_{i, \mathrm{fl}}(\vec{q})=\alpha_{s}^{i}(\vec{q}) \vec{v}_{i}(\vec{q}) e^{-i \omega_{s} t}+\alpha_{\mathrm{idler}}^{i}(\vec{q})\left[\vec{v}_{j}(\vec{Q}-\vec{q})\right]^{*} e^{i \omega_{\mathrm{idler}} t},
$$

where $j$ and $\vec{Q}$ are determined by the matrix $M_{1}$. In particular, $\vec{Q}$ corresponds to the momentum of the pump. For effective Hamiltonians considered in the main text in Eqs. (40) and (41), the Floquet eigenstates involve a linear combination of two equilibrium states. The coefficients $\left\{\alpha_{s}^{i}(\vec{q}), \alpha_{\text {idler }}^{i}(\vec{q})\right\}$ 
obey the secular equation

$$
\left(\begin{array}{cc}
-i\left[\omega_{s}-\omega_{i}(\vec{q})\right] & i g^{*} \\
-i g & i\left[\omega_{\text {idler }}-\omega_{j}(\vec{q}-\vec{Q})\right]
\end{array}\right) \cdot\left(\begin{array}{c}
\alpha_{s}^{i}(q) \\
\alpha_{\text {idler }}^{i}(q)
\end{array}\right)=0,
$$

where $g$ is proportional to the amplitude of the oscillating field and the matrix element of the overlap with matrix $M_{1}$. Solution of (E5) is found by setting the determinant to zero:

$$
\left[\omega_{s}-\omega_{i}(\vec{q})\right]\left[\omega_{\text {idler }}-\omega_{j}(\vec{q}-\vec{Q})\right]=|g|^{2} .
$$

Equation (E6) provides an implicit equation for the wave vector $\vec{q}$ of the transmitted wave when the incident beam oscillates at the signal frequency $\omega_{s}$. In the normal incidence geometry considered in this paper (see Fig. 7), $q_{z}=0$. For small enough $|g|$, the above equation has two solutions, a signal solution $q_{s}$ for which $\omega_{i}\left(q_{s}\right) \approx \omega_{s}$ and an idler $q_{\text {idler }}$ for which $\omega_{j}\left(Q-q_{\text {idler }}\right) \approx \omega_{\text {idler }}$. The distinction is blurred close to parametric resonance when $\omega_{i}(q)+\omega_{j}(Q-q)=\Omega_{\mathrm{fl}}$ and thus the two solutions will be denoted as $q=q_{ \pm}$. From the secular equation, the Floquet eigenstate corresponding to the transmitted wave is given by

$$
v_{\mathrm{fl}, \mathrm{i}}\left(q_{ \pm}\right)=E_{0} t_{ \pm}^{i}\left(g \vec{v}_{i}(\vec{q})+\left[\omega_{s}-\omega_{i}(\vec{q})\right]\left[\vec{v}_{j}(\vec{Q}-\vec{q})\right]^{*}\right)
$$

where we set $\quad \alpha_{s}^{i}\left(\vec{q}_{ \pm}\right)=E_{0} t_{ \pm}^{i} g, \quad \alpha_{\text {idler }}^{i}\left(\vec{q}_{ \pm}\right)=$ $E_{0} t_{ \pm}^{i}\left[\omega_{s}-\omega_{i}\left(\vec{q}_{ \pm}\right)\right]$. The overall normalization of the Floquet eigenstate is absorbed in the transmission coefficients $\left\{t_{ \pm}^{i}\right\}$ for each transmission channel and $E_{0}$ is the amplitude of the incident electric field. The explicit form of the electric potential in the transmitted wave is given by

$$
\begin{aligned}
V_{\lambda, i}(\vec{x}, t)= & E_{0} \sum_{l} t_{ \pm}^{l}\left(\alpha_{s}^{l}\left(\vec{q}_{ \pm}^{l}\right) v_{l}^{V_{\lambda, i}}\left(\vec{q}_{ \pm}^{l}\right) e^{i q_{ \pm}^{l} x} e^{-i \omega_{s} t}+\alpha_{\mathrm{idler}}^{l}\left(\vec{q}_{ \pm}^{l}\right)\right. \\
& \left.\times\left(v_{l^{\prime}}^{V_{\lambda, i}}\left(\vec{Q}-\vec{q}_{ \pm}^{l}\right)\right)^{*} e^{i\left(q_{ \pm}^{l}-Q_{x}\right) x} e^{-i Q_{z} D i} e^{i \omega_{\mathrm{ideler}} t}\right)
\end{aligned}
$$

and similarly for $\vec{A}_{\vec{x}}$ and $A_{z}$. In Eq. (E8), different independent transmission channels are included explicitly denoted by $l$, where transmission coefficients and wave vectors depend on the particular transmission channel (see discussion of the static case in Sec. II D).

As in the static case the transmission electric and magnetic fields are calculated through Eq. (29), repeated here for convenience:

$$
\begin{aligned}
& E_{z, \lambda, i}=-\Delta_{z} V_{\lambda, i}-\partial_{t} A_{z, \lambda, i}, \\
& B_{z, \lambda, i}=-\partial_{x} A_{z, \lambda, i}+\Delta_{z} A_{x, \lambda, i} .
\end{aligned}
$$

Since the transmitted waves have components that oscillate at the idler frequency due to the Floquet drive, the reflected wave necessarily has components that also oscillate at the idler frequency to match boundary conditions. The out-of-plane momentum of the drive along the $c$ axis is conserved across the interface. The idler boundary conditions are given by

$$
\begin{aligned}
& r_{\omega_{\text {idler }}, Q_{z}+\frac{2 \pi n}{D}} E_{0}=E_{t, \omega_{\text {idler }}, Q_{z}}+\frac{2 \pi n}{D}, \\
& -\frac{i \sqrt{\left(Q_{z}+\frac{2 \pi n}{D}\right)^{2}-\frac{\omega_{i \text { iller }}^{2}}{c_{v a c}^{2}}}}{\omega_{\text {idler }}} r_{\omega_{\text {idler }}, Q_{z}+\frac{2 \pi n}{D}} E_{0}=B_{t, \omega_{\text {ider }}, Q_{z}+\frac{2 \pi n}{D}},
\end{aligned}
$$

where we included the possibility for Bragg reflection as in equilibrium (discussed in Sec. IID). Schematically, the reflected and transmitted waves in a Floquet medium are shown in Fig. 10.

Finally, we note that one could simplify the system of equations even further by including only the transmitted channel corresponding to modes with the most overlap with the incoming beam (lower JP for signal frequencies $\omega_{s}<10 \mathrm{THz}$ and upper JP for signal frequencies $\omega_{s}>12 \mathrm{THz}$ ). Consequently, to keep the number of variables the same as the boundary conditions, one needs to assume that Bragg reflection of the light is negligible and consider only the $n=0$ case in Eqs. (E10) and (26). This approximation is valid when one of the transmission channels is either heavily localized at the interface as an evanescent wave within the frequency range considered or has a large in-plane current density component and hence small overlap with the electric field.

\section{Dissipation and causality}

Within our formalism, phenomenological dissipation is added through the dispersion relation by replacing $\omega_{s} \rightarrow \omega_{s}+$ $i \gamma_{i}(q)$ where dissipation can depend on the particular transmission channel. Note that the above recipe implies for the idler frequency $\omega_{\text {idler }} \rightarrow \omega_{\text {idler }}-i \gamma_{i}(q)$. Even in the absence of dissipation, an infinitesimal decay constant is needed to unambiguously determine the direction of the transmitted wave. The wave-vector solutions to the Floquet-Fresnel problem $q_{ \pm}$ are chosen such that the (possibly infinitesimal) imaginary part is positive and therefore decays away from the surface and into the material. This procedure shows that the solution corresponding to the idler mode propagates toward the surface. This underlies parametric amplification, where energy can be extracted from the oscillating field in the form of waves transmitted outside of the material. The above situation is depicted schematically in Fig. 10.
[1] A. von Hoegen, M. Fechner, M. Först, J. Porras, B. Keimer, M. Michael, E. Demler, and A. Cavalleri, Probing coherent charge fluctuations in $\mathrm{YBa}_{2} \mathrm{Cu}_{3} \mathrm{O}_{6+x}$ at wavevectors outside the light cone, arXiv:1911.08284.

[2] W. Hu, S. Kaiser, D. Nicoletti, C. R. Hunt, I. Gierz, M. C. Hoffmann, M. Le Tacon, T. Loew, B. Keimer, and A. Cavalleri, Optically enhanced coherent transport in $\mathrm{YBa}_{2} \mathrm{Cu}_{3} \mathrm{O}_{6.5}$ by ultrafast redistribution of interlayer coupling, Nat. Mater. 13, 705 (2014).

[3] S. Kaiser, C. R. Hunt, D. Nicoletti, W. Hu, I. Gierz, H. Y. Liu, M. Le Tacon, T. Loew, D. Haug, B. Keimer, and A. Cavalleri, Optically induced coherent transport far above $T_{c}$ in underdoped $\mathrm{YBa}_{2} \mathrm{Cu}_{3} \mathrm{O}_{6+\delta}$, Phys. Rev. B 89, 184516 (2014). 
[4] D. Fausti, R. I. Tobey, N. Dean, S. Kaiser, A. Dienst, M. C. Hoffmann, S. Pyon, T. Takayama, H. Takagi, and A. Cavalleri, Light-induced superconductivity in a stripe-ordered cuprate, Science 331, 189 (2011).

[5] D. Nicoletti, E. Casandruc, Y. Laplace, V. Khanna, C. R. Hunt, S. Kaiser, S. S. Dhesi, G. D. Gu, J. P. Hill, and A. Cavalleri, Optically induced superconductivity in striped $\mathrm{La}_{2-x} \mathrm{Ba}_{x} \mathrm{CuO}_{4}$ by polarization-selective excitation in the near infrared, Phys. Rev. B 90, 100503(R) (2014).

[6] S. J. Zhang, Z. X. Wang, L. Y. Shi, T. Lin, M. Y. Zhang, G. D. Gu, T. Dong, and N. L. Wang, Light-induced new collective modes in the superconductor $\mathrm{La}_{1.905} \mathrm{Ba}_{0.095} \mathrm{CuO}_{4}$, Phys. Rev. B 98, 020506(R) (2018).

[7] K. A. Cremin, J. Zhang, C. C. Homes, G. D. Gu, Z. Sun, M. M. Fogler, A. J. Millis, D. N. Basov, and R. D. Averitt, Photoenhanced metastable c-axis electrodynamics in stripe-ordered cuprate $\mathrm{La}_{1.885} \mathrm{Ba}_{0.115} \mathrm{CuO}_{4}$, Proc. Natl. Acad. Sci. USA 116, 19875 (2019).

[8] M. Budden, T. Gebert, M. Buzzi, G. Jotzu, E. Wang, T. Matsuyama, G. Meier, Y. Laplace, D. Pontiroli, M. Riccó, F. Schlawin, D. Jaksch, and A. Cavalleri, Evidence for metastable photo-induced superconductivity in $\mathrm{K}_{3} \mathrm{C}_{60}$, arXiv:2002.12835.

[9] M. Buzzi, D. Nicoletti, M. Fechner, N. Tancogne-Dejean, M. A. Sentef, A. Georges, M. Dressel, A. Henderson, T. Siegrist, J. A. Schlueter, K. Miyagawa, K. Kanoda, M. S. Nam, A. Ardavan, J. Coulthard, J. Tindall, F. Schlawin, D. Jaksch, and A. Cavalleri (unpublished).

[10] N. Dasari and M. Eckstein, Transient floquet engineering of superconductivity, Phys. Rev. B 98, 235149 (2018).

[11] M. Babadi, M. Knap, I. Martin, G. Refael, and E. Demler, Theory of parametrically amplified electron-phonon superconductivity, Phys. Rev. B 96, 014512 (2017).

[12] Y. Murakami, N. Tsuji, M. Eckstein, and P. Werner, Nonequilibrium steady states and transient dynamics of conventional superconductors under phonon driving, Phys. Rev. B 96, 045125 (2017).

[13] J. ichi Okamoto, W. Hu, A. Cavalleri, and L. Mathey, Transiently enhanced interlayer tunneling in optically driven high- $T_{c}$ superconductors, Phys. Rev. B 96, 144505 (2017).

[14] S. J. Denny, S. R. Clark, Y. Laplace, A. Cavalleri, and D. Jaksch, Proposed Parametric Cooling of Bilayer Cuprate Superconductors by Terahertz Excitation, Phys. Rev. Lett. 114, 137001 (2015).

[15] D. M. Kennes, M. Claassen, M. A. Sentef, and C. Karrasch, Light-induced $d$-wave superconductivity through floquet - engineered fermi surfaces in cuprates, Phys. Rev. B 100, 075115 (2019)

[16] Z. M. Raines, V. Stanev, and V. M. Galitski, Enhancement of superconductivity via periodic modulation in a three-dimensional model of cuprates, Phys. Rev. B 91, 184506 (2015).

[17] A. A. Patel and A. Eberlein, Light-induced enhancement of superconductivity via melting of competing bond-density wave order in underdoped cuprates, Phys. Rev. B 93, 195139 (2016).

[18] N. Bittner, T. Tohyama, S. Kaiser, and D. Manske, Possible light-induced superconductivity in a strongly correlated electron system, J. Phys. Soc. Jpn. 88, 044704 (2019).

[19] G. Chiriacò, A. J. Millis, and I. L. Aleiner, Transient superconductivity without superconductivity, Phys. Rev. B 98, 220510(R) (2018).
[20] J. Demsar, Non-equilibrium phenomena in superconductors probed by femtosecond time-domain spectroscopy, J. Low Temp. Phys. (2020), doi: 10.1007/s10909-020-02461-y.

[21] Z. Sun and A. J. Millis (unpublished).

[22] Y. Lemonik and A. Mitra, Transport and spectral signatures of transient fluctuating superfluids in the absence of long-range order, Phys. Rev. B 100, 094503 (2019).

[23] M. A. Sentef, A. Tokuno, A. Georges, and C. Kollath, Theory of Laser-Controlled Competing Superconducting and Charge Orders, Phys. Rev. Lett. 118, 087002 (2017).

[24] M. A. Sentef, A. F. Kemper, A. Georges, and C. Kollath, Theory of light-enhanced phonon-mediated superconductivity, Phys. Rev. B 93, 144506 (2016).

[25] Y. J. Uemura, L. P. Le, G. M. Luke, B. J. Sternlieb, W. D. Wu, J. H. Brewer, T. M. Riseman, C. L. Seaman, M. B. Maple, M. Ishikawa, D. G. Hinks, J. D. Jorgensen, G. Saito, and H. Yamochi, Basic Similarities among Cuprate, Bismuthate, Organic, Chevrel-Phase, and Heavy-Fermion Superconductors Shown by Penetration-Depth Measurements, Phys. Rev. Lett. 66, 2665 (1991).

[26] V. J. Emery and S. A. Kivelson, Importance of phase fluctuations in superconductors with small superfluid density, Nature (London) 374, 434 (1995).

[27] Q. Chen, J. Stajic, S. Tan, and K. Levin, Bcs - bec crossover: From high temperature superconductors to ultracold superfluids, Phys. Rep. 412, 1 (2005).

[28] V. M. Loktev, R. M. Quick, and S. G. Sharapov, Phase fluctuations and pseudogap phenomena, Phys. Rep. 349, 1 (2001).

[29] Z. Tešanović, O. Vafek, and M. Franz, Chiral symmetry break-

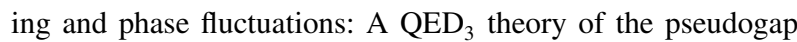
state in cuprate superconductors, Phys. Rev. B 65, 180511(R) (2002).

[30] Y. Wang, Z. A. Xu, T. Kakeshita, S. Uchida, S. Ono, Y. Ando, and N. P. Ong, Onset of the vortexlike nernst signal above $T_{c}$ in $\mathrm{La}_{2-x} \mathrm{Sr}_{x} \mathrm{CuO}_{4}$ and $\mathrm{Bi}_{2} \mathrm{Sr}_{2-y} \mathrm{La}_{y} \mathrm{CuO}_{6}$, Phys. Rev. B 64, 224519 (2001).

[31] D. Podolsky, S. Raghu, and A. Vishwanath, Nernst Effect and Diamagnetism in Phase Fluctuating Superconductors, Phys. Rev. Lett. 99, 117004 (2007).

[32] L. Li, Y. Wang, S. Komiya, S. Ono, Y. Ando, G. D. Gu, and N. P. Ong, Diamagnetism and cooper pairing above $T_{c}$ in cuprates, Phys. Rev. B 81, 054510 (2010).

[33] B. Keimer, S. A. Kivelson, M. R. Norman, S. Uchida, and J. Zaanen, From quantum matter to high-temperature superconductivity in copper oxides, Nature (London) 518, 179 (2015).

[34] P. Zhou, L. Chen, Y. Liu, I. Sochnikov, A. T. Bollinger, M.G. Han, Y. Zhu, X. He, I. Bozovic, and D. Natelson, Electron pairing in the pseudogap state revealed by shot noise in copper oxide junctions, Nature (London) 572, 493 (2019).

[35] A. E. Koshelev and L. N. Bulaevskii, Fluctuation broadening of the plasma resonance in the vortex liquid state of layered superconductors, Phys. Rev. B 60, R3743(R) (1999).

[36] H. Won and K. Maki, d-wave superconductor as a model of high- $t_{c}$ superconductors, Phys. Rev. B 49, 1397 (1994).

[37] P. Monthoux and G. G. Lonzarich, p-wave andd-wave superconductivity in quasi-two-dimensional metals, Phys. Rev. B 59, 14598 (1999).

[38] Y. K. Kim, N. H. Sung, J. D. Denlinger, and B. J. Kim, Observation of a d-wave gap in electron-doped $\mathrm{Sr}_{2} \mathrm{IrO}_{4}$, Nat. Phys. 12, 37 (2016). 
[39] R. Kleiner, P. Muller, and S. Sakai, Dynamic behavior of Josephson coupled layered structures, Phys. Rev. B 50, 3942 (1994).

[40] L. N. Bulaevskii, M. Zamora, D. Baeriswyl, H. Beck, and J. R. Clem, Time-dependent equations for phase differences and a collective mode in josephson-coupled layered superconductors, Phys. Rev. B 50, 12831 (1994).

[41] L. Bulaevskii, D. Domínguez, M. Maley, A. Bishop, O. K. C. Tsui, and N. Ong, Linewidth of c-axis plasma resonance in Josephson-coupled superconductors, Phys. Rev. B 54, 7521 (1996).

[42] A. E. Koshelev, L. N. Bulaevskii, and M. P. Maley, Josephson coupling, phase correlations, and Josephson plasma resonance in vortex liquid phase, Phys. Rev. B 62, 14403 (2000).

[43] D. van der Marel and A. A. Tsvetkov, Transverse-optical josephson plasmons: Equations of motion, Phys. Rev. B 64, 024530 (2001).

[44] T. Koyama and M. Tachiki, I-v characteristics of josephsoncoupled layered superconductors with longitudinal plasma excitations, Phys. Rev. B 54, 16183 (1996).

[45] T. Koyama, Josephson plasma resonances and optical properties in high-tc superconductors with alternating junction parameters, J. Phys. Soc. Jpn. 71, 2986 (2002).

[46] J. ichi Okamoto, A. Cavalleri, and L. Mathey, Theory of Enhanced Interlayer Tunneling in Optically Driven High - $T_{c}$ Superconductors, Phys. Rev. Lett. 117, 227001 (2016).

[47] Y. Laplace and A. Cavalleri, Josephson plasmonics in layered superconductors, Adv. Phys.: X 1, 387 (2016).

[48] S. Sugiura, E. A. Demler, M. Lukin, and D. Podolsky (unpublished).

[49] M. Buzzi, G. Jotzu, A. Cavalleri, J. Ignacio Cirac, E. A. Demler, B. I. Halperin, M. D. Lukin, T. Shi, Y. Wang, and D. Podolsky (unpublished).

[50] S. Rajasekaran, E. Casandruc, Y. Laplace, D. Nicoletti, G. D. Gu, S. R. Clark, D. Jaksch, and A. Cavalleri, Parametric amplification of a superconducting plasma wave, Nat. Phys. 12, 1012 (2016).

[51] A. Eckardt and E. Anisimovas, High-frequency approximation for periodically driven quantum systems from a floquet - space perspective, New J. Phys. 17, 093039 (2015).

[52] F. Schackert, A. Roy, M. Hatridge, M. H. Devoret, and A. D. Stone, Three-Wave Mixing with Three Incoming Waves: Signal-Idler Coherent Attenuation and Gain Enhancement in a Parametric Amplifier, Phys. Rev. Lett. 111, 073903 (2013).

[53] Note that we use notations for the interlayer currents which are convenient in the discussion of lattice QED but differ from the usual notations in superconductivity. The interlayer current in our notation corresponds to the physical current times the appropriate layer separation, which explains the extra factors of $1 / d_{\lambda}$ in Eq. (4) and factors $d_{\lambda}^{2}$ in Eq. (2).

[54] S. N. Gupta, Theory of longitudinal photons in quantum electrodynamics, Proc. Phys. Soc. A 63, 681 (1950).

[55] K. Bleuler, A new method of treatment of the longitudinal and scalar photons, Helv. Phys. Acta 23, 567 (1950).

[56] M. wen Xiao (unpublished).

[57] M. Tinkham, Introduction to Superconductivity (Dover, New York, 2004).

[58] Intuitively off-resonant contributions average to zero. Within perturbation theory off-resonant corrections are divided by their detuning, making such contributions subleading [59].

[59] M. R. Vega, M. Lentz, and B. Seradjeh, Floquet perturbation theory: formalism and application to low - frequency limit, New J. Phys. 20, 093022 (2018).

[60] P. Savidis, J. J. Baumberg, R. M. Stevenson, M. S. Skolnick, D. M. Whittaker, and J. S. Roberts, Angle Resolved Stimulated Photonic Amplifier, Phys. Rev. Lett. 84, 1547 (2000).

[61] L. N. Bulaevskii, M. Ledvij, and V. G. Kogan, Vortices in layered superconductors with Josephson coupling, Phys. Rev. B 46, 366 (1992).

[62] A. E. Koshelev, Plasma Resonance and Remaining Josephson Coupling in the "Decoupled Vortex Liquid Phase" in Layered Superconductors, Phys. Rev. Lett. 77, 3901 (1996).

[63] Y. J. Uemura, G. M. Luke, B. J. Sternlieb, J. H. Brewer, J. F. Carolan, W. N. Hardy, R. Kadono, J. R. Kempton, R. F. Kiefl, S. R. Kreitzman, P. Mulhern, T. M. Riseman, D. Ll. Williams, B. X. Yang, S. Uchida, H. Takagi, J. Gopalakrishnan, A. W. Sleight, M. A. Subramanian, C. L. Chien et al., Universal Correlations between $T_{c}$ and $\frac{n_{s}}{m^{*}}$ (Carrier Density Over Effective Mass) in High- $T_{c}$ Cuprate Superconductors, Phys. Rev. Lett. 62, 2317 (1989).

[64] A. Paramekanti, M. Randeria, T. V. Ramakrishnan, and S. S. Mandal, Effective actions and phase fluctuations in d-wave superconductors, Phys. Rev. B 62, 6786 (2000).

[65] L. Benfatto, S. Caprara, C. Castellani, A. Paramekanti, and M. Randeria, Phase fluctuations, dissipation, and superfluid stiffness in d-wave superconductors, Phys. Rev. B 63, 174513 (2001).

[66] B. Meng, M. Singleton, M. Shahmohammadi, F. Kapsalidis, R. Wang, M. Beck, and J. Faist, Mid-infrared frequency comb from a ring quantum cascade laser, Optica 7, 162 (2020).

[67] J. S. Schalch, K. Post, G. Duan, X. Zhao, Y. D. Kim, J. Hone, M. M. Fogler, X. Zhang, D. N. Basov, and R. D. Averitt, Strong metasurface-Josephson plasma resonance coupling in superconducting $\mathrm{La}_{(2-x)} \mathrm{Sr}_{\mathrm{x}}-\mathrm{CuO}_{4}$, Adv. Opt. Mater. 7, 1900712 (2019). 